\title{
INTERGALACTIC TRANSMISSION AND ITS IMPACT ON THE LY $\alpha$ LINE
}

\author{
Peter Laursen ${ }^{1,2}$, Jesper Sommer-Larsen ${ }^{1,3,4}$ and Alexei O. Razoumov ${ }^{5}$ \\ 2nd draft
}

\begin{abstract}
We study the intergalactic transmission of radiation in the vicinity of the Ly $\alpha$ wavelength. Simulating sightlines through the intergalactic medium (IGM) in detailed cosmological hydrosimulations, the impact of the IGM on the shape of the line profile from Ly $\alpha$ emitting galaxies at redshifts 2.5 to 6.5 is investigated. In particular we show that taking into account the correlation of the density and velocity fields of the IGM with the galaxies, the blue part of the spectrum may be appreciably reduced, even at relatively low redshifts. This may in some cases provide an alternative to the often-invoked outflow scenario, although it is concluded that this model is still a plausible explanation of the many asymmetric Ly $\alpha$ profiles observed.

Applying the calculated wavelength dependent transmission to simulated spectra from Ly $\alpha$ emitting galaxies, we derive the fraction of photons that are lost in the IGM, in addition to what is absorbed internally in the galaxies due to dust.

Moreover, by comparing the calculated transmission of radiation blueward of the Ly $\alpha$ line with corresponding observations, we are able to constrain the epoch when the Universe was reionized to $z \lesssim 8.5$
\end{abstract}

Subject headings: intergalactic medium — radiative transfer — scattering — line: profiles

\section{INTRODUCTION}

The past decade has seen a rapid increase in the use of $\mathrm{Ly} \alpha$ as a cosmological tool. A plethora of physical characteristics of young galaxies and the intergalactic medium (IGM) that separate them manifest themselves in the shape, the strength, and the spatial distribution of both Ly $\alpha$ emission and absorption lines.

Since generally the evolution of astrophysical objects happen on timescales much longer than a human lifetime, numerical simulations are particularly well-suited for exploring the Universe. Many elaborate numerical codes have been constructed to model the Universe on all scales, from dust agglomeration, over planetary and stellar formation, to simulations of cosmological volumes. Although these codes include a steadily increasing number of physical processes, numerical resolution, etc., and although they are able to predict various observables, many of them fail to account for the fact that the observed light may be rather different from the light that was emitted. Once radiation leaves its origins, it may still be subject to various physical processes altering not only its intensity, but in some cases also its spatial and spectral distribution. If we do not understand these processes, we may severely misinterpret the predictions of the models when comparing to observations. This is particularly true with regard to resonant lines like Ly $\alpha$,

\footnotetext{
${ }^{1}$ Dark Cosmology Centre, Niels Bohr Institute, University of Copenhagen, Juliane Maries Vej 30, DK-2100, Copenhagen $\varnothing$, Denmark; email: pela@dark-cosmology.dk, j.sommerlarsen@gmail.com.

2 Oskar Klein Centre, Dept. of Astronomy, Stockholm University, AlbaNova, SE-10691 Stockholm, Sweden.

${ }^{3}$ Excellence Cluster Universe, Technische Universität München, Boltzmannstraße 2, 85748 Garching, Germany.

${ }^{4}$ Marie Kruses Skole, Stavnsholtvej 29-31, DK-3520 Farum, Denmark.

5 SHARCNET/UOIT, 2000 Simcoe Street N., Oshawa ON L1H7K4, Canada; email: razoumov@sharcnet.ca.
}

where its complicated path may take it through regions of unknown physical conditions. In order to interpret the observed Ly $\alpha$ lines correctly, it is crucial to have an understanding of the physical processes that influence the shape and transmission of the line.

Although the atomic processes regulating the individual scatterings are always the same ${ }^{6}$, the physical conditions governing different regions in space will have a large impact on the outcome. For example, photons produced by gravitational cooling are mostly born in low-density regions, and may escape the galaxy more or less freely. These photons thus tend to have a frequency close to the line center. On the other hand, photons produced in the dense stellar regions will usually have to scatter far from the line center in order to escape, and thus tend to comprise the wings of the spectrum. However, these photons are also more vulnerable to dust, since their paths are much longer, and since also most of the galactic dust resides in these emission regions. Moreover, the velocity field of the gas elements also has an effect on the observed line profile. In particular macroscopic gas motions, such as gas accretion or outflows caused by feedback due to massive star formation, will skew the line (e.g. Dijkstra et al. 2006; Verhamme et al. 2006).

While the history of theoretical Ly $\alpha$ radiative transfer (RT) dates back to the 1930's (Ambarzumian 1932; Chandrasekhar 1935), the first practical prediction of spectra emerging from a source embedded in neutral hydrogen was given by Harrington (1973), and later generalized by Neufeld (1990). Due to the high opacity for a photon at the line center, in general the photons have to diffuse in frequency and should hence escape the medium in a double-peaked spectrum. Although this

\footnotetext{
${ }^{6}$ As long the atom is not perturbed during the $\sim 10^{-8}$ seconds it is excited; this, however, can be safely ignored in most astrophysical situations.
} 
has indeed been observed (e.g. Yee \& De Robertis 1991; Venemans et al. 2005; Vanzella 2008), apparently most Ly $\alpha$ profiles from high-redshift galaxies seem to be missing the blue peak. An immediate conclusion would be that high-redshift, Ly $\alpha$ emitting galaxies (LAEs) are in the process of massive star formation and thus exhibit strong outflows. Indeed, this scenario has been invoked to explain a large number of LAE spectra, most convincingly by Verhamme et al. (2008) who, assuming a central source and thin surrounding shell of neutral gas while varying its expansion velocity, temperature, and gas and dust column density, manage to produce nice fits to a number of observed spectra.

Although the thin expanding shell scenario hinges on a physically plausible mechanism, it is obviously rather idealized. Furthermore, since most observations show only the red peak of the profile, it seems to indicate that most (high-redshift) galaxies exhibit outflows. However, at high redshifts many galaxies are still forming, resulting in infall which would in turn imply an increased blue peak. Since this is rarely observed, in this paper we aim to investigate whether some other mechanism, such as IGM RT effects, could be responsible for removing the blue peak and/or enhancing the red peak.

The present study complements other recent endeavors to achieve a comprehensive understanding of how the Ly $\alpha$ line is redistributed in frequency and real space after having escaped its host galaxy (Iliev et al. 2008; Barnes \& Haehnelt 2010; Faucher-Giguère et al. 2010; Zheng et al. 2010a,b,c). Our cosmological volume is not as large as most of these studies, and although gas dynamics are included in the simulations, ioniziation by UV radiation is calculated as a post-process rather than on the fly. The reward is a highly increased resolution, allowing us to study the circumgalactic environs in great detail. Moreover, we inquire into the temporal evolution of the IGM.

The exploration of the high-redshift $\operatorname{Ly} \alpha$ Universe is rapidly progressing, gradually providing a census of the physical properties of young galaxies, such as their luminosity functions, clustering properties, evolution, and contents. Recent notable surveys have revealed hundreds of LAEs at high redshifts, from $z \sim 2.1-2.3$ (Guaita et al. 2010; Nilsson et al. 2009), to $z \sim 4.5$ (Wang et al. 2009), to $z \sim 6.6$ (Ouchi et al. 2010), and for small samples even farther (Hibon et al. 2010; Tilvi et al. 2010), and the present work, with its emphasis on the reshaping of the Ly $\alpha$ line profile and amplitude throughout different epochs is a step toward a more accurate interpretation of the observations.

In the following section, we discuss qualitatively the impact of the IGM on the Ly $\alpha$ line profile. In Section 3 we then decribe the numerical simulations we have performed to study the effect quantitatively, the results of which are presented in Section 4. We discuss our findings in Section 5, and summarize in Section 6.

\section{THE INTERGALACTIC MEDIUM}

As light travels through the expanding Universe, it gets redshifted, implying that wavelengths $\lambda$ blueward of the Ly $\alpha$ line center are eventually shifted into resonance. If, for a given wavelength, this happens in the vicinity of a sufficient amount of neutral hydrogen, the spectrum experiences an absorption line (although strictly speaking the photons are not absorbed, but rather scattered out of the line of sight). This results in the so-called Ly $\alpha$ forest (LAF; Lynds 1971; Sargent et al. 1980).

As the red part of the spectrum is only shifted farther away from resonance, IGM absorption tends to skew the line and not simply diminish it by some factor. In most earlier studies of the line profiles of highredshift galaxies, the IGM has either been ignored (e.g. Verhamme et al. 2006, 2008), taken to transmit the red half and remove the blue part (e.g. Finkelstein et al. 2008), or to influence the line uniformly by a factor $e^{-\langle\tau\rangle}$, where $\langle\tau\rangle$ is the average optical depth of the Universe (e.g. Bruscoli et al. 2003; Meiksin 2005; Faucher-Giguère et al. 2008; Rykoff et al. 2009).

Consider a source emitting the normalized spectrum $F_{\text {em }}(\lambda) \equiv 1$ for all $\lambda$. In an idealized, completely homogeneous universe undergoing completely homologous expansion ("ideal Hubble flow") and with the absorption profiles having a negligible width, the observed spectrum would simply be a step function, with $F(\lambda)=F_{\text {red }}=1$ for $\lambda>\lambda_{0}$, and $F(\lambda)=F_{\text {blue }}<1$ for $\lambda \leq \lambda_{0}$, where $\lambda_{0}=1216 \AA$ is the central wavelength of the line. Three factors contribute to make $F$ differ from a step function:

Firstly, the Ly $\alpha$ line is not a delta function, but has a finite width, in some cases extending significantly into the red part of the spectrum. Secondly, the IGM is highly inhomogeneous, leading to large variations in $F$ blueward of $\lambda_{0}$ - the LAF. If we consider the average effect of the IGM and let $F$ be the average of many sightlines, $F_{\text {blue }}$ should still be a constant function of wavelength. However, in the proximity of galaxies the gas density is higher than far from the galaxies; on the other hand, in these regions the stellar ionizing UV radiation may reduce the neutral gas fraction. Consequently, wavelengths just blueward of the line center may not on average be subject to the same absorption as farther away from the line. Regardless of which of the two effects - gas overdensity or stronger UV field - is more important, the correlation of the IGM with the source cannot be neglected.

Finally the expansion is not exactly homologous, since peculiar velocities of the gas elements will cause fluctuations around the pure Hubble flow. Considering again not individual sightlines but the average effect of the IGM, these fluctuations are random and cancel out; on average, for every gas element that recedes from a galaxy faster than the Hubble flow and thus causes an absorption line at a slightly bluer wavelength, another gas element does the opposite. Hence, the average transmission in a "realistic" universe is the same as in a universe where there are no peculiar velocities. However, in the proximity of overdensities, the extra mass results in a "retarded" expansion of the local IGM. When expansion around a source is somewhat slower than that of the rest of the Universe, on average matter in a larger region will be capable of causing absorption, since the slower the expansion, the farther the photons will have to travel before shifting out of resonance.

\subsection{The transmission function}

This paper focuses on two different aspects of the intergalactic transmission. First, to see how the Ly $\alpha$ line is affected by the IGM, we calculate a "transmission func- 
tion" given by the average, normalized flux $F(\lambda)$. Specifically, $F$ is calculated by taking the median value in each wavelength bin of many sightlines, originating just outside a large number of galaxies (where "just outside" will be defined later). The standard deviation is defined by the 16 and 84 percentiles. From the above discussion we may expect that $F$ be characterized by a red part $F_{\text {red }} \simeq 1$, and a blue part $F_{\text {blue }}<1$, but with a nontrivial shape just blueward of the line center, since the IGM in the vicinity of the sources is different than far from the sources.

\subsection{The average transmission}

We will also examine the average transmission $\mathcal{T}=$ $\mathcal{T}(z)$ of the IGM, defined by first calculating for each individual sightline the fraction of photons that are transmitted through the IGM in a relatively large wavelength interval well away from the line center, and then taking the median of all sightlines (again with the 16 and 84 percentiles defining the standard deviation). This quantity is sensitive to the overall ionization state of the IGM, and has therefore been used observationally to put constraints on the so-called Epoch of Reionization (EoR; e.g. Becker et al. 2001; Djorgovski et al. 2001). A paramount puzzle in modern cosmology is the question of when and how the hydrogen, and later helium, was reionized. The EoR marks a comprehensive change of the physical state of the gaseous Universe, and to understand the cause, as well as the course, of this phenomenon is a challenging task. Besides being a compelling event in itself, it also has profound implications for the interpretation of observations and theoretical cosmological models, not only due to the increased transparency of the IGM, but also because of the accompanying rise in IGM temperature.

One way of probing the EoR is by looking at the spectra of high-redshift quasars. The intrinsic spectrum of a quasar is characterized by broad emission lines; in particular the Ly $\alpha$ line is very prominent. For larger redshifts the absorption lines of the LAF gets increasingly copious, until eventually all of the light blueward of $\operatorname{Ly} \alpha$ is absorbed, resulting in the so-called Gunn-Peterson trough (Gunn \& Peterson 1965). Observations of a large number of quasars show that the Universe was largely opaque to radiation blueward of Ly $\alpha$ at $z \gtrsim 6$ (Songaila 2004; Fan et al. 2006).

\section{SIMULATIONS}

Laursen et al. (2009a,b) considered numerically the shape of the Ly $\alpha$ spectrum emerging from galaxies at $z \sim 3.5$, based on hydro/gravity galaxy simulations of very high resolution. In these studies, the RT was carried out with Monte Carlo simulations, following individual photons (or photon packets) as they scatter stochastically out through the interstellar medium. Since high resolution is important for the $\operatorname{Ly} \alpha \mathrm{RT}$, especially when taking into account dust, the simulations were conducted in galaxies extracted from a fully cosmological, large volume model, resimulated ${ }^{7}$ at high resolution, and interpolated onto an adaptively refined grid. This Ly $\alpha$ RT

7 A "resimulation" is performed by tracing the position of the particles comprising a given galaxy back to the initial conditions, and then repeating the simulation at 8 or 64 times the original mass resolution. evidently only predicts the spectrum of radiation that one would observe if located in the vicinity of the galaxies. In reality the radiation has to travel trough the IGM. At a redshift of $\sim 3.5$, the IGM is largely ionized, and the spectra escaping the galaxies are, in general, not very different from what would be observed at Earth. However, even a very small amount of neutral hydrogen may influence the observations. To investigate just how large an impact the IGM exerts on the radiation, full IGM RT has to be computed. Furthermore, at higher redshifts where the IGM is generally more neutral and more dense, neglecting the effect would lead to severely erroneous results.

In principle this could be achieved by performing first the "galactic" RT in the high-resolution resimulations and subsequently continuing the RT in the low-resolution cosmological volume from the location of the individual galaxies. However, although the physics of scattering in galaxies and that of scattering in the IGM is not inherently different, the difference in physical conditions imposes a natural division of the two schemes: in the dense gas of galaxies, photons are continuously scattered in and out of the line of sight, whereas in the IGM, once a photon is scattered out of the line of sight, it is "lost", becoming part of the background radiation. The probability of a background photon being scattered into the line of sight, on the other hand, is vanishingly small.

In order to disentangle galactic from intergalactic effects, and, more importantly, to investigate the general effect of the IGM instead of merely the IGM lying between us and a handful of resimulated galaxies, we take a different approach: the transmission properties of the IGM are studied by calculating the normalized spectrum $F(\lambda)$ - the transmission function - in the vicinity of the Ly $\alpha$ line, as an average of a large number of sightlines cast through the cosmological volume, and originating just outside a large number of galaxies.

\subsection{Cosmological simulations}

A standard, flat $\Lambda \mathrm{CDM}$ is assumed, with $\Omega_{m}=0.3$, $\Omega_{\Lambda}=0.7$, and $h=H_{0} / 100 \mathrm{~km}^{-1} \mathrm{Mpc}^{-1}=0.7$.

The radiative transfer is carried out in a cosmological volume modeled using self-consistent, $a b$ initio hydro/gravity simulations (Sommer-Larsen et al. 2003; Sommer-Larsen 2006). The code used in these simulations is a significantly improved version of the TreeSPH code, which has been used previously for galaxy formation simulations (Sommer-Larsen et al. 2003). The main improvements over the previous version are: (1) The "conservative" entropy equation solving scheme (Springel \& Hernquist 2002) has been implemented, improving shock resolution in SPH simulations. In particular, this improves the resolution of the starburst-driven galactic superwinds invoked in the simulations. (2) Noninstantaneous gas recycling and chemical evolution, tracing the ten elements $\mathrm{H}, \mathrm{He}, \mathrm{C}, \mathrm{N}, \mathrm{O}, \mathrm{Mg}, \mathrm{Si}, \mathrm{S}, \mathrm{Ca}$ and Fe (Lia et al. 2002a,b); the algorithm invokes effects of supernovae of type II and type Ia, and mass loss from stars of all masses. (3) Atomic radiative cooling depending both on the metal abundance of the gas and on the meta-galactic UV background (UVB), modeled after Haardt \& Madau (1996), is invoked, as well as a simplified treatment of radiative transfer, switching off the UVB where the gas becomes optically thick to Ly- 
man limit photons on a scale of $0.1 \mathrm{kpc}$ (Sommer-Larsen 2006). The simple scheme for UVB RT is supplemented by a more elaborate post-process ionizing UV RT scheme, as described in Section 3.2.

The simulation volumes were selected from two dark matter (DM) only cosmological simulations of box length $10 h^{-1} \mathrm{Mpc}$, set up at $z_{i}=39$ using identical Fourier modes and phases, but with rms linear fluctuations on a scale of $8 h^{-1} \mathrm{Mpc}, \sigma_{8}$, equal to either 0.74 or 0.9 , respectively, bracketing the latest $\mathrm{WMAP}^{8}$-inferred value of $\sim 0.8$ (Jarosik et al. 2010).

The original DM-only simulations were run with periodic boundary conditions using $128^{3}$ DM particles, and have been used previously for galaxy resimulations (e.g. Sommer-Larsen et al. 2003; Sommer-Larsen 2006, note, however, that in these simulations, $\sigma_{8}=1.0$ was assumed). For each of the two cosmological DM-only simulations, mass and force resolution was increased (by factors of eight and two, respectively) in a spherical Lagrangian sub-volume of radius $5 h^{-1} \mathrm{Mpc}$ (about half the volume of the original DM-only simulation), and in this region all DM particles were split into a DM particle and a gas (SPH) particle according to an adopted universal baryon fraction of $f_{b}=0.15$, in line with recent estimates. The cosmological regions resimulated thus had a comoving volume of about $1500 \mathrm{Mpc}^{3}$, contained about 17 million particles in total, and were resimulated with an open boundary condition using the full hydro/gravity code. The numerical resolution of these cosmological resimulations was hence the same as that of the individual galaxy formation simulations of Sommer-Larsen et al. (2003), reflecting the very considerable increase in computing power since then.

The masses of SPH, star and DM particles were $m_{\text {gas }}=$ $m_{\star}=7.3 \times 10^{5}$ and $m_{\mathrm{DM}}=4.2 \times 10^{6} h^{-1} \mathrm{M}_{\odot}$, and the gravity softening lengths were $\epsilon_{\text {gas }}=\epsilon_{\star}=380$ and $\epsilon_{\mathrm{DM}}=680 h^{-1} \mathrm{pc}$. The gravity softening lengths were kept constant in physical coordinates from $z=6$ to $z=$ 0 , and constant in comoving coordinates at earlier times.

The choice of stellar initial mass function (IMF) affects the chemical evolution and the energetics of stellar feedback processes, in particular starburst-driven galactic superwinds. Motivated by the findings of Sommer-Larsen \& Fynbo (2008), a Salpeter (1955) IMF was adopted for the simulations presented in this paper.

\subsection{Ionizing $U V$ radiative transfer}

The Haardt \& Madau UVB field becomes negligible at $z \geq z_{\text {re }}=6$. To comply with the results of WMAP, which indicate a somewhat earlier onset of the ionizing background, we ran an additional set of models, in which the UVB intensity curve was "stretched" to initiate at $z_{\mathrm{re}}=10$. In the following, Model 1, 2, and 3 refers to simulations using $\left(z_{\mathrm{re}}, \sigma_{8}\right)=(10,0.74),(10,0.9)$, and $(6,0.74)$, respectively. The models (also summarized in Table 1) with $z_{\mathrm{re}}=10$ will be referred to as "early" reionization, while $z_{\text {re }}=6$ will be referred to as "late" reionization. In Sections 4.1 and 4.3 we study the differences between these models.
A realistic UV RT scheme (Razoumov \& Sommer-Larsen 2006, 2007) was em-

8 http://lambda.gsfc.nasa.gov/product/map/current/ ployed to post-process the results of the hydrodynamical simulations using the following approach: First, the physical properties of the SPH particles (density, temperature, etc.) are interpolated onto an adaptively refined grid of base resolution $128^{3}$, with dense cells recursively subdivided into eight cells, until no cell contains more than ten particles. Around each stellar source, a number of radial rays are constructed and followed through the nested grid, accumulating photoreaction number and energy rates in each cell. Rays are split either as one moves farther away from the source, or as a refined cell is entered. These reaction rates are used to compute iteratively the equilibrium ionization state of each cell.

In addition to stellar photons, we also account for ionization and heating by $\mathrm{LyC}$ photons originating outside the computational volume with the FTTE scheme (Razoumov \& Cardall 2005) assuming the Haardt \& Madau UVB, modified to match the particular reionization model.

Since the relative ratio of $\mathrm{HI}$, HeI, and HeII densities varies from cell to cell, the UV RT is computed separately in three frequency bands, [13.6, 24.6[ eV, $[24.6,54.4[\mathrm{eV}$, and $[54.4, \infty[\mathrm{eV}$, respectively. In each cell the angleaveraged intensity is added to the chemistry solver to compute the ionization equilibrium.

\subsection{Galaxy selection criteria}

Galaxies are located in the simulations as described in Sommer-Larsen et al. (2005). To make sure that a given identified structure is a real galaxy, the following selection criteria are imposed on the sample:

1. To ensure that a given structure " $i$ " is not just a substructure of a larger structure " $j$ ", if the center of structure $i$ is situated within the virial radius of $j$, it must have more stars than $j$.

2. The minimum number of star particles must be at least $N_{\star, \min }=15$. This corresponds to a minimum stellar mass of $\log \left(M_{\star, \min } / M_{\odot}\right)=7.2$.

3. The circular velocity, given by $V_{c}=\sqrt{G M_{\mathrm{vir}} / r_{\mathrm{vir}}}$ must be $V_{c} \geq 35 \mathrm{~km} \mathrm{~s}^{-1}$.

The IGM in the vicinity of large galaxies is to some extend different from the IGM around small galaxies. The more mass gives rise to a deeper gravitational potential, enhancing the retarded Hubble flow. On the other hand, the larger star formation may cause a larger bubble of ionized gas around it. To investigate the difference in transmission, the sample of accepted galaxies is divided into three subsamples, denoted "small", "intermediate", and "large". To use the same separating criteria at all redshifts, instead of separating by mass - which increases with time due to merging and accretion - the galaxies are separated according to their circular velocity, which does not change significantly over time. The thresholds are defined somewhat arbitrarily as $V_{1}=55$ $\mathrm{km} \mathrm{s}^{-1}$ (between small and intermediate galaxies) and $V_{2}=80 \mathrm{~km} \mathrm{~s}^{-1}$ (between intermediate and large galaxies). The final sample of galaxies is shown in Figure 1, which shows the relation between stellar and virial mass, and the distribution of circular velocities. 

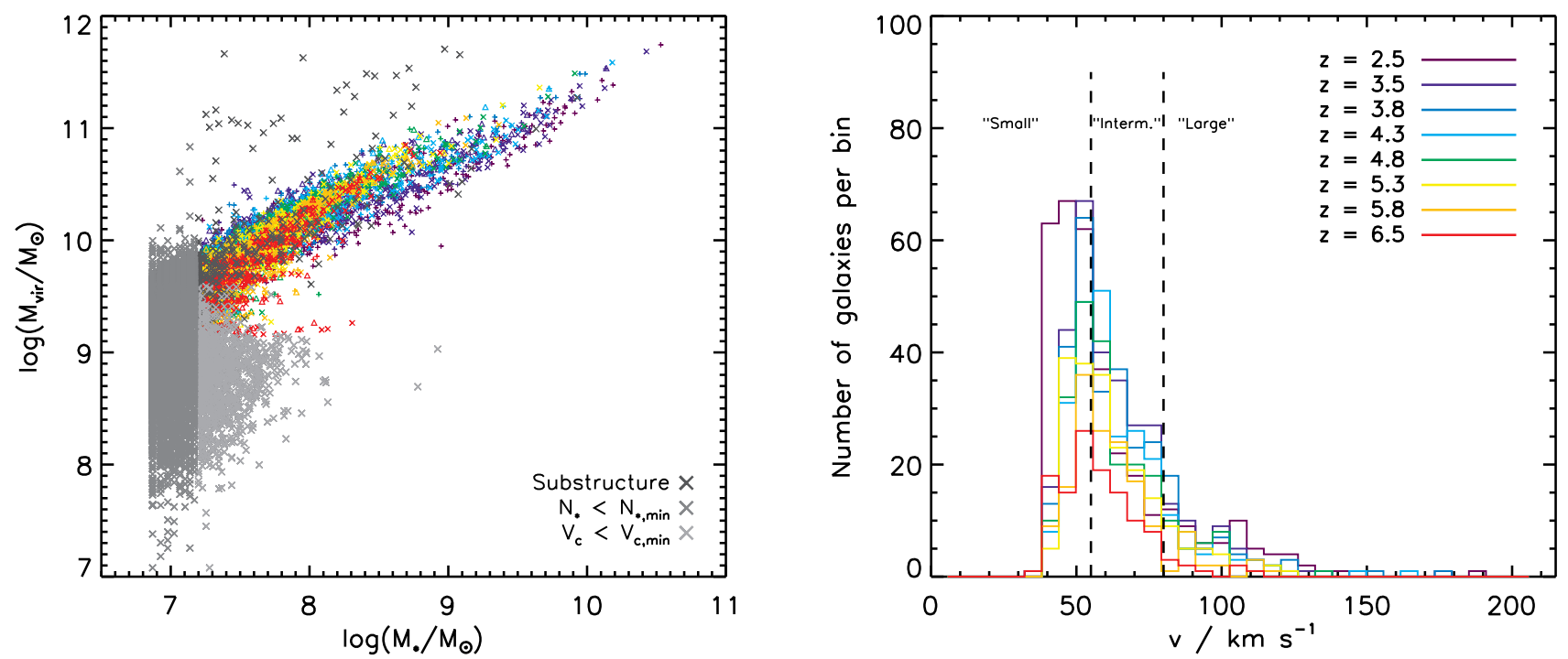

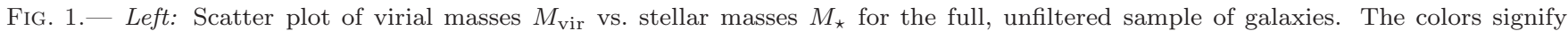
redshift, with more red meaning higher redshift (exact values are seen in the right plot's legend). The data points of Model 1, 2, and 3 are shown with triangles, plus signs, and crosses, respectively. The galaxies that are rejected are overplottet with gray crosses, with dark, medium, and light gray corresponding to rejection criterion 1,2, and 3, respectively. Right: Distribution of circular velocities $V_{c}$ for the accepted galaxies in Model 1. The distributions for Model 2 and 3 look similar, although Model 2 has more galaxies (see Table 1).

TABLE 1

Number of galaxies in the Simulations

\begin{tabular}{cccccccc}
\hline \hline Model & $z_{\text {re }}$ & $\sigma_{8}$ & $z$ & Total & Small & Intermediate & Large \\
& & & & & & & \\
\hline 1. & 10 & 0.74 & 2.5 & 343 & 207 & 80 & 56 \\
& & & 3.5 & 309 & 138 & 127 & 44 \\
& & & 3.8 & 283 & 125 & 119 & 39 \\
& & & 4.3 & 252 & 102 & 115 & 35 \\
& & & 5.8 & 225 & 111 & 86 & 28 \\
& & & 5.8 & 154 & 98 & 85 & 18 \\
& & & 6.5 & 121 & 70 & 62 & 17 \\
2. & \multirow{2}{*}{10} & 0.9 & 3.5 & 405 & 207 & 128 & 70 \\
& & & 4.3 & 384 & 165 & 150 & 69 \\
& & & 4.8 & 341 & 150 & 129 & 62 \\
& & & 5.3 & 324 & 145 & 125 & 54 \\
& & & 6.5 & 252 & 121 & 106 & 50 \\
3. & 6 & 0.74 & 3.5 & 325 & 162 & 92 & 34 \\
& & & 3.8 & 318 & 165 & 122 & 41 \\
& & 4.3 & 293 & 150 & 110 & 36 \\
& & 4.8 & 250 & 138 & 84 & 28 \\
& & 5.3 & 204 & 101 & 85 & 18 \\
& & 5.8 & 160 & 75 & 67 & 18 \\
& & & 6.5 & 126 & 69 & 50 & 7 \\
\end{tabular}

Note. — "Small", "intermediate", and "large" galaxies are defined as having circular velocities $V_{c}<55 \mathrm{~km} \mathrm{~s}^{-1}, 55 \mathrm{~km}$ $\mathrm{s}^{-1} \leq V_{c}<80 \mathrm{~km} \mathrm{~s}^{-1}$, and $V_{c} \geq 80 \mathrm{~km} \mathrm{~s}^{-1}$, respectively.'

The exact number of galaxies in the three models studied in this work is seen in Table 1.

\subsection{IGM radiative transfer}

The IGM RT is conducted using the code IGMTRANS$\mathrm{FER}^{9}$. For the RT we use the same nested grid used for

9 The code can be downloaded from the following URL: http: //www. dark-cosmology.dk/ pela/IGMtransfer.html. the ionizing UV RT. The transmission properties of the IGM are studied by calculating the normalized spectrum $F(\lambda)$ in the vicinity of the Ly $\alpha$ line, as an average of a high number of sightlines cast through the simulated cosmological volume.

The resulting value of $F(\lambda)$ at wavelength $\lambda$ for a given sightline is

$$
F(\lambda)=e^{-\tau(\lambda)}
$$

The optical depth $\tau$ is the sum of contributions from all the cells encountered along the line of sight:

$$
\tau(\lambda)=\sum_{i}^{\text {cells }} n_{\mathrm{HI}, i} r_{i} \sigma\left(\lambda+\lambda v_{\|, i} / c\right) .
$$

Here, $n_{\mathrm{HI}, i}$ is the density of neutral hydrogen in the $i$ 'th cell, $r_{i}$ is the distance covered in that particular cell, $v_{\|, i}$ is the velocity component of the cell along the line of sight, and $\sigma(\lambda)$ is the cross section of neutral hydrogen. Due to the resonant nature of the transition, the largest contribution at a given wavelength will arise from the cells the velocity of which corresponds to shifting the wavelength close to resonance.

Although no formal definition of the transition from a galaxy to the IGM exists, we have to settle on a definition of where to begin the sightlines, i.e. the distance $r_{0}$ from the center of a galaxy. Observed Ly $\alpha$ profiles result from scattering processes in first the galaxy and subseqently the IGM, and regardless of the chosen value of $r_{0}$, for consistency "galactic Ly $\alpha$ RT" should be terminated at the same value when coupling the two RT schemes. In galactic Ly $\alpha$ RT, individual photons are traced, while in IGM RT we only consider the photons that are not scattered out of the line of sight. Hence the sightlines should begin where photons are mainly scattered out of the line of sight, and only a small fraction is scattered into the line of sight. Since more neutral gas is associated with larger galaxies, as well as with higher redshift, clearly 
$r_{0}$ depends on the galaxy and the epoch, but measuring $r_{0}$ in units of the virial radius $r_{\text {vir }}$ helps to compare the physical conditions around different galaxies. In Section 4.1 we argue the a reasonable value of $r_{0}$ is $1.5 r_{\text {vir }}$, and show that the final results are only mildly sensitive to the actual chosen value of $r_{0}$.

At a given redshift, $F(\lambda)$ is calculated as the median in each wavelength bin of $10^{3}$ sightlines from each galaxy in the sample (using $10^{4}$ sightlines produces virtually identical results). The number of galaxies amounts to several hundreds, and increases with time (cf. Table 1). The simulated volume is a spherical region of comoving diameter $D_{\text {box }}$, but to avoid any spuriosities at the edge of the region only a $0.9 D_{\text {box }}$ sphere is used. Due to the limited size of the volume, in order to perform the RT until a sufficiently short wavelength is redshifted into resonance the sightlines are allowed to "bounce" within the sphere, such that a ray reaching the edge of the sphere is re"emitted" back in a random angle in such a way that the total volume is equally well sampled. Note however that in general the wavelength region close to the line that is affected by the correlation of the IGM with the source is reached well before the the first bouncing.

The normalized spectrum is emitted at rest wavelength in the reference frame of the center of mass of a galaxy, which in turn may have a peculiar velocity relative to the cell at which it is centered. This spectrum is then Lorentz transformed between the reference frames of the cells encountered along the line of sight. Since the expansion of space is approximately homologous, each cell can be perceived as lying in the center of the simulation, and hence this bouncing scheme does not introduce any bias, apart from reusing the same volume several times for a given sightline. However, since the sightlines scatter around stochastically and thus pierce a given region from various directions, no periodicities arise in the calculated spectrum.

To probe the average transmission, the sightlines are propagated until the wavelength $1080 \AA$ has been redshifted into resonance, corresponding to $\Delta z \simeq 0.1$. In this case the sightlines bounce roughly 30 (20) times at $z=2.5(6.5)$.

\section{RESULTS}

In the following sections, Model 1 is taken to be the "benchmark" model, while the others are discussed in Section 5.5.

\subsection{The Ly $\alpha$ transmission function}

Figure 2 shows the calculated transmission functions $F(\lambda)$ at various redshifts. Indeed, a dip just blueward of the line center is visible at all redshifts. The results in Figure 2 were calculated as the median of sightines emerging from all galaxies in the sample. Of course a large scatter exists, since each sightline goes through very different regions, even if emanating from the same galaxy. Figure 3 shows the scatter associated with $F$ for three different redshifts. The equivalent transmission functions for the $z_{\mathrm{re}}=6$ model are shown in Figure 4 . While at high redshifts the $z_{\mathrm{re}}=6$ model clearly results in a much more opaque universe, at lower redshifts the transmission properties of the IGM in the different models are more similar, although at $z=3.5$, the $z_{\text {re }}=10$ model still transmits more light.
To see the difference in transmission around galaxies of different sizes, Figure 5 shows transmission function for three size ranges (defined in Section 3.3). Since the distance from the galaxies at which the sightlines start is given in terms of virial radii, sightlines emerging from small galaxies start closer to their source than for large galaxies, and since at lower redshifts they tend to be clustered together in the same overdensities as large galaxies, this results in slightly more absorption. However, the difference is not very significant.

\subsection{Effect on the spectrum and escape fraction}

In Figure 6 the "purpose" of the transmission function is illustrated: the left panel shows the spectrum emerging from a galaxy of $M_{\mathrm{vir}}=4.9 \times 10^{9} M_{\odot}$ and Ly $\alpha$ luminosity $L_{\mathrm{Ly} \alpha}=4.9 \times 10^{40} \mathrm{erg} \mathrm{s}^{-1}$, calculated with the Monte Carlo Ly $\alpha$ RT code MoCALATA (Laursen et al. 2009a). Its circular velocity of $42 \mathrm{~km} \mathrm{~s}^{-1}$ characterizes it as a small galaxy. The spectrum which is actually observed, after the light has been transferred through the IGM, is shown in the right panel. Although on average the effect of the IGM is not very large at this redshift, as seen by the solid line in the right panel, due to the large dispersion in transmission (visualized by the gray-shaded area) at least some such galaxies will be observed with a substantially diminished blue peak.

In general, the larger a galaxy is the broader its emitted spectrum is, since Ly $\alpha$ photons have to diffuse farther from the line center for higher column densities of neutral gas. If dust is present, this will tend to narrow the line (Laursen et al. 2009b). Larger galaxies tend to have higher metallicities and hence more dust, but the lines will still be broader than the ones of small galaxies. The galaxy used in Figure 6 is quite small. In Figure 7 we show the impact of the IGM on the nine simulated spectra from Laursen et al. (2009b), spanning a range in stellar masses from $\sim 10^{7}$ to $\sim 10^{10}$. For comparison, typical LAEs have stellar masses of $M_{\star} / M_{\odot} \sim 10^{8}-10^{9}$ (Gawiser et al. 2006; Lai et al. 2007; Finkelstein et al. 2007; Nilsson et al. 2007, obtained from SED fitting).

Besides altering the shape of the emitted spectrum, the IGM also has an effect on another quantity of much interest to observers, namely the observed fraction $f_{\text {obs }}$ of the intrinsically emitted number of Ly $\alpha$ photons. Since the bulk of the emitted Ly $\alpha$ photons is due to young stars, the total Ly $\alpha$ luminosity of a galaxy may be used as a proxy for its star formation rate (SFR), one of the main quantities characterizing galaxies. Assuming case B recombination, $L_{\mathrm{Ly} \alpha}$ can be converted to a total $\mathrm{H} \alpha$ luminosity $L_{\mathrm{H} \alpha}$ (through $L_{\mathrm{H} \alpha}=L_{\mathrm{Ly} \alpha} / 8.7$; Osterbrock 1989), which in turn can be converted to an SFR (through SFR $=7.9 \times 10^{-42} L_{\mathrm{H} \alpha} /\left(\mathrm{erg} \mathrm{s}^{-1}\right) M_{\odot} \mathrm{yr}^{-1}$; Kennicutt 1998).

The above conversion factors assume that none of the emitted light is lost. If dust is present in the galaxy a fraction of the emitted photons will be absorbed (possibly making the LAE observable in FIR; see Dayal et al. 2010a). This can be corrected for if the color excess $E(B-V)$ is measured, assuming some standard extinction curve. However, this assumes that both the $\mathrm{H} \alpha$ and Ly $\alpha$ radiation are simply reduced by some factor corresponding to having traveled the same distance through the dusty medium. But since the path of Ly $\alpha$ photons is increased by resonant scattering, this may be far from 

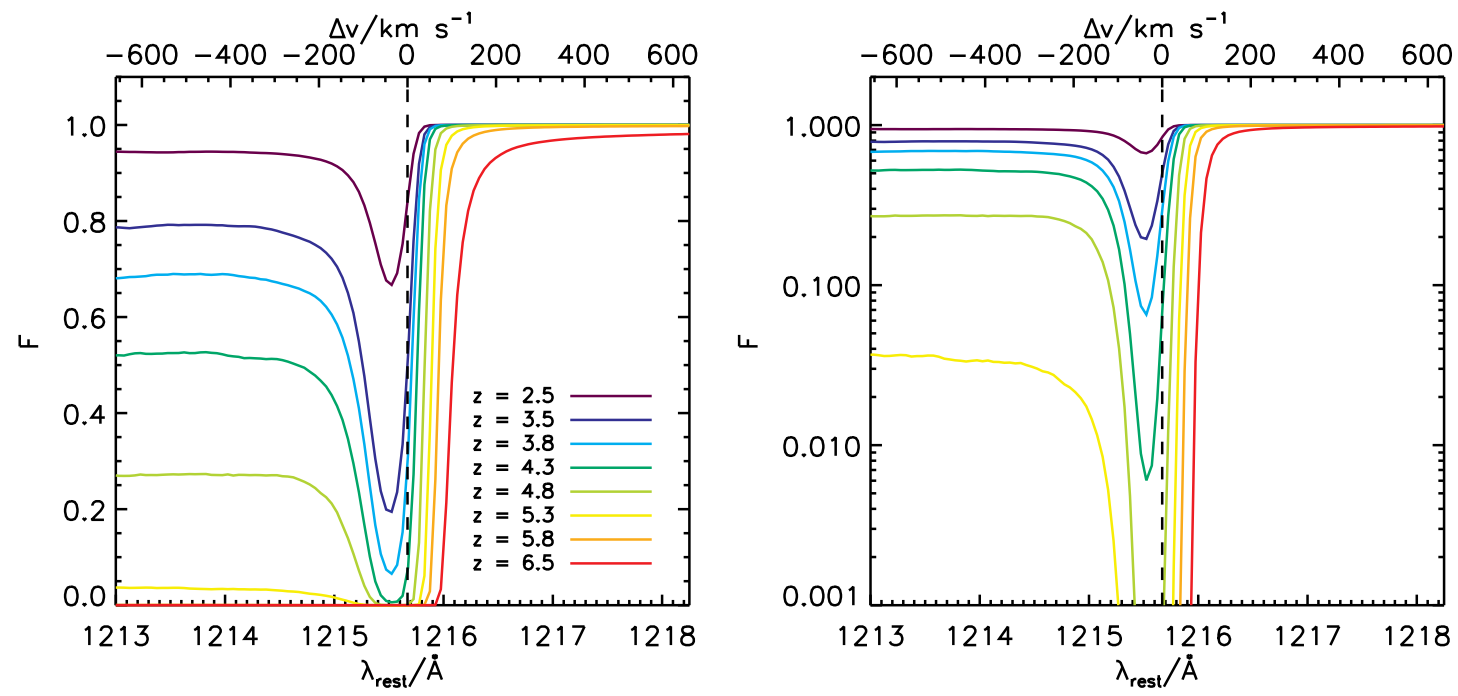

FIG. 2.- Normalized transmission $F(\lambda)$ at wavelengths around Ly $\alpha$, for different redshifts given by the color. In the left panel, the vertical axis is linear, while in the right it is logarithmic, emphasizing the transmission at high redshifts.
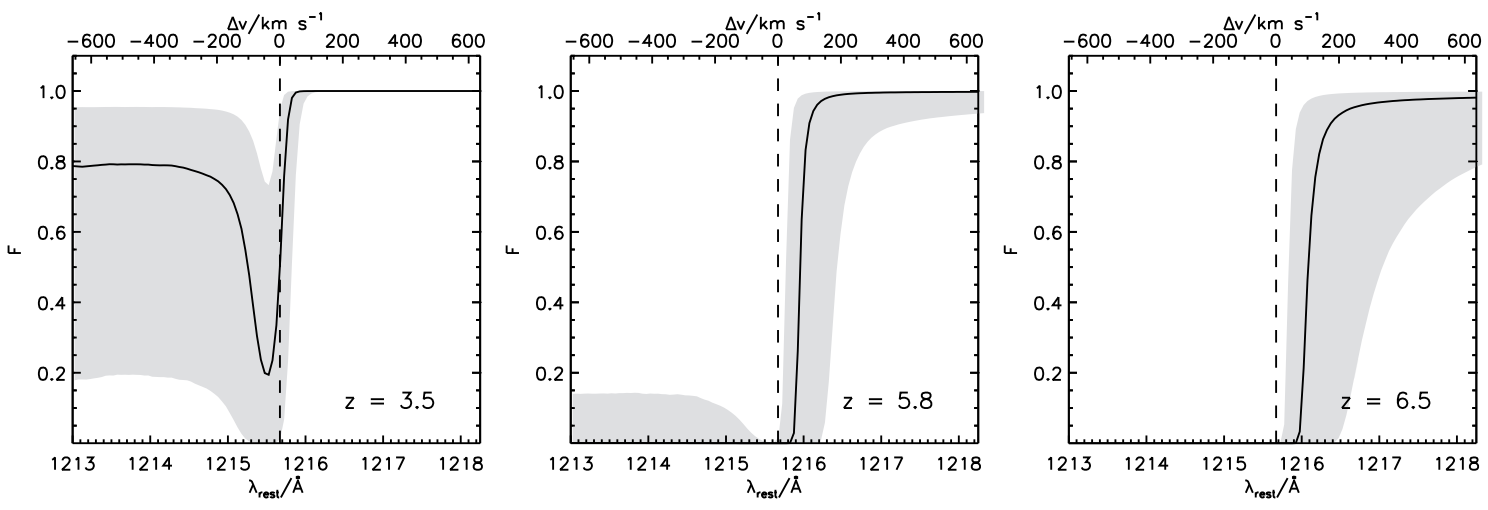

Fig. 3. - Transmission $F$ for $z=3.5$ (left), 5.8 (middle), and 6.5 (right) in Model 1, i.e. with $\sigma_{8}=0.74$ and $z_{\text {re }}=10$. The shaded region indicates the range within which $68 \%$ of the individual calculated transmission functions fall.
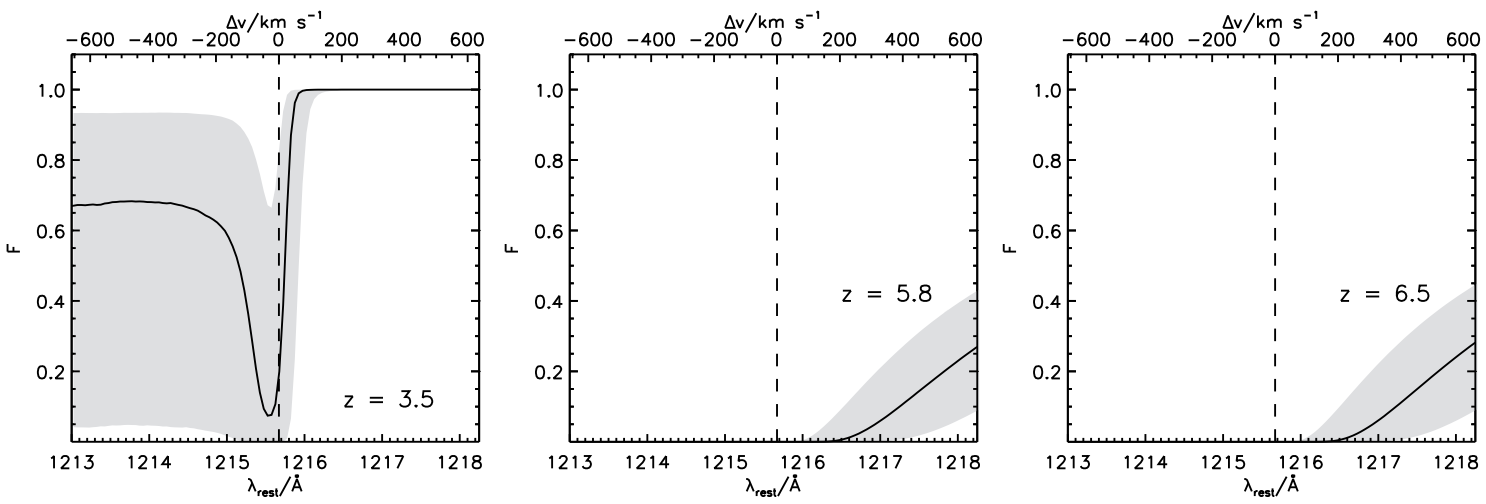

FIG. 4. - Same as Figure 3 for Model 3, i.e. with $z_{\mathrm{re}}=6$. While at high redshifts a much more neutral IGM than in the $z_{\mathrm{re}}=10$ model causes a severe suppression of the Ly $\alpha$ line, by $z=3.5$ the state of the IGM is not very different. 

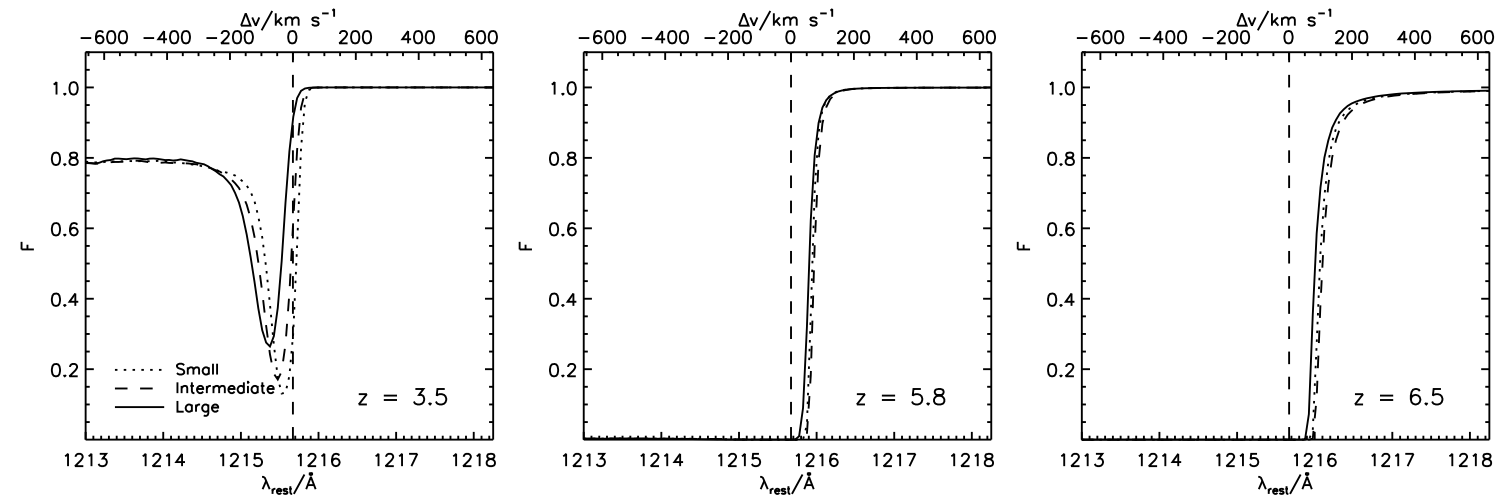

Fig. 5.- Transmission $F$ for $z=3.5$ (left), 5.8 (middle), and 6.5 (right), for three different size categories of galaxies; small (dotted), intermediate (dashed), and large (solid lines). Although slightly more absorption is seen in the vicinity of smaller galaxies, the transmission functions are quite similar for the three size ranges.
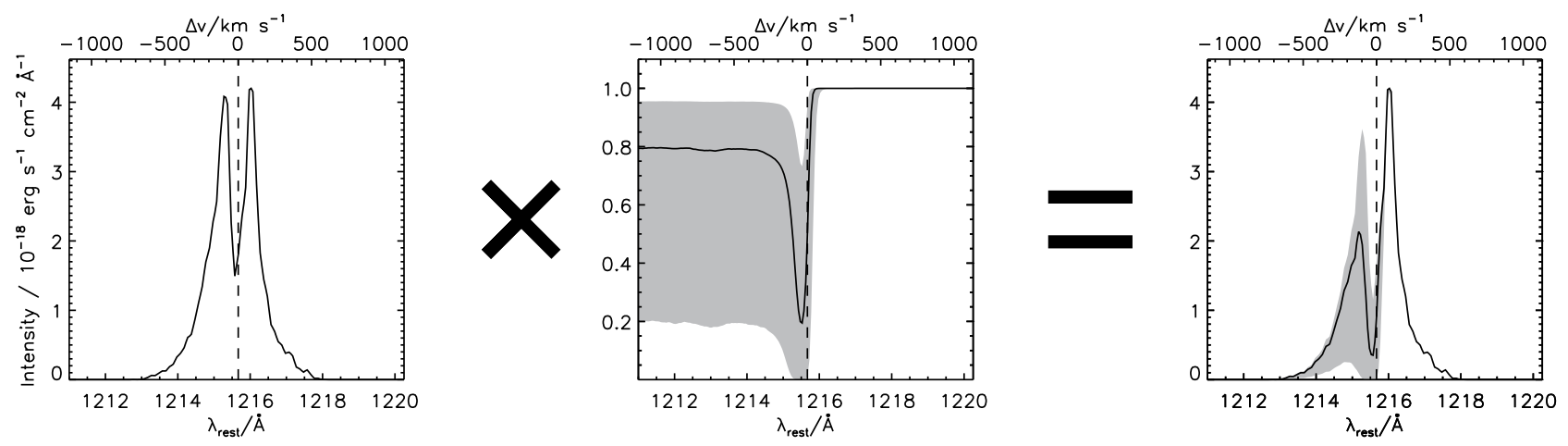

FIG. 6. - Illustration of the effect of the IGM on the observed Ly $\alpha$ profile emerging from a galaxy at $z \sim 3.5$. Without taking into account the IGM the two peaks are roughly equally high (left panel). However, when the spectrum is transmitted through the IGM characterized by the transmission function $F(\lambda)$ (middle panel), the blue peak is dimished, resulting in an observed spectrum with a higher red peak (right panel). 


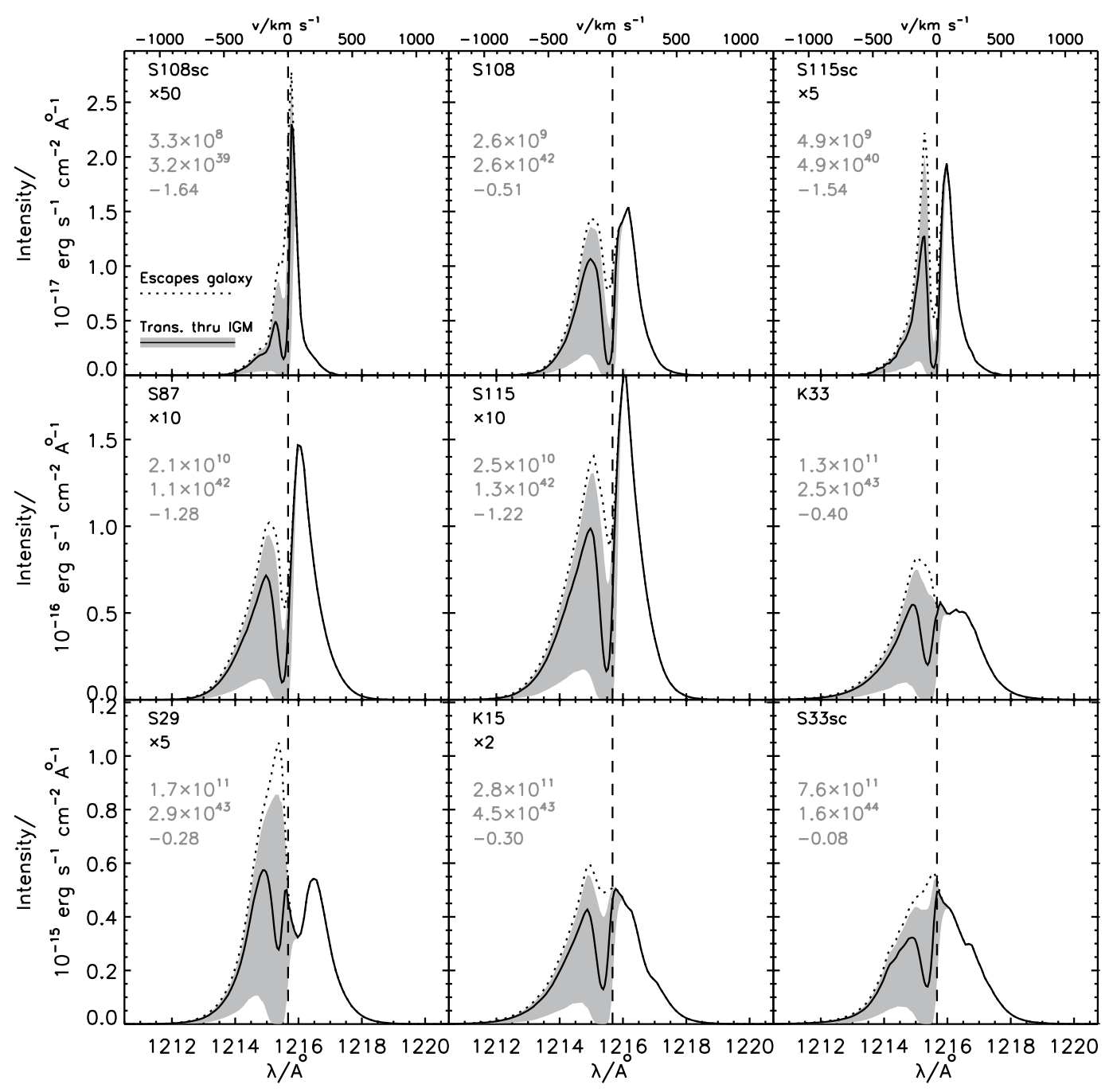

FIG. 7.- Emitted spectra (dotted lines) from nine different simulated galaxies at $\sim 3.5$ - ordered after increasing size - and the corresponding spectra after being transmitted through the IGM (solid lines with gray regions denoting the $68 \%$ confidence intervals). The transmission functions appropriate for the given galaxy sizes have been used. In order to use the same ordinate axis for a given row, some intensities have been multiplied a factor indicated under the name of the galaxy. The numbers shown in gray are the corresponding galaxy's virial mass in $M_{\odot}$, its Ly $\alpha$ luminosity in $\mathrm{erg} \mathrm{s}^{-1}$, and its oxygen metallicity $[\mathrm{O} / \mathrm{H}]$, respectively.

the truth. Comparing the Ly $\alpha$-inferred SFR with that of $\mathrm{H} \alpha$ (or UV continuum), the effect of scattering can be constrained, as the quantity $\mathrm{SFR}(\mathrm{Ly} \alpha) / \mathrm{SFR}(\mathrm{H} \alpha ; \mathrm{UV})$ will be an estimate of $f_{\text {obs }}$. Using this technique, values from a few percent (mostly in the nearby Universe; e.g. Hayes et al. 2007; Atek et al. 2008; Hayes et al. 2010) to $\sim 1 / 3$ at high redshifts (Gronwall et al. 2007) have been found.

Once the radiation enters the IGM, it is also not affected in the same way, since the IGM is transparent to $\mathrm{H} \alpha$, but not to Ly $\alpha$. Dijkstra et al. (2007) estimated analytically the fraction of Ly $\alpha$ photons that are scattered out of the line of sight by the IGM (at $z \sim 4.5-6.5$ ) and found a mean transmission of $f_{\mathrm{IGM}} \sim 0.1-0.3$, depending on various assumptions (note that in Dijkstra et al. (2007) this fraction is called " $\mathcal{T}_{\alpha}$ "). In that study, the intrinsic Ly $\alpha$ line profile is modeled as a Gaussian, the width of which is given by the circular velocity of the galaxies, in turn given by their mass, and assuming that no dust in the galaxies alters the shape of the line before the light enters the IGM.

Since the transmission function is a non-trivial function of wavelength, the exact shape and width of the Ly $\alpha$ line profile is important. With the Ly $\alpha$ RT code MoCALATA more realistic spectra can be modeled, and applying the transmission function found in Section 4.1, $f_{\text {IGM }}$ can be calculated for the sample of simulated galaxies. The transmitted fraction is not a strong function of galaxy size; on average, a fraction $f_{\mathrm{IGM}}=0.77_{-0.34}^{+0.17}$ of the photons escaping the galaxies is transmitted through the IGM, at the investigated redshift of $z \sim 3.5$. The results for the individual galaxies is given in Table 2 .

At higher redshifts the metallicity is generally lower, 
TABLE 2

LY $\alpha$ TRANSMISSION FRACTIONS FOR NINE SIMULATED GALAXIES AT $z \sim 3.5$

\begin{tabular}{|c|c|c|c|c|}
\hline Galaxy & $V_{c}$ & $f_{\text {esc }}$ & $f_{\mathrm{IGM}}$ & $f_{\text {obs }}$ \\
\hline S108sc & 17 & $0.97 \pm 0.02$ & $0.66_{-0.36}^{+0.24}$ & $0.64_{-0.35}^{+0.23}$ \\
\hline S108 & 33 & $0.12 \pm 0.02$ & $0.78_{-0.35}^{+0.16}$ & $0.09_{-0.04}^{+0.02}$ \\
\hline $\mathrm{S} 115 \mathrm{sc}$ & 42 & $0.95 \pm 0.03$ & $0.76_{-0.34}^{+0.18}$ & $0.72_{-0.32}^{+0.17}$ \\
\hline S87 & 69 & $0.22 \pm 0.01$ & $0.81_{-0.32}^{+0.15}$ & $0.18_{-0.07}^{+0.03}$ \\
\hline $\mathrm{S} 115$ & 73 & $0.30 \pm 0.05$ & $0.80_{-0.34}^{+0.16}$ & $0.24_{-0.11}^{+0.06}$ \\
\hline K33 & 126 & $0.08 \pm 0.02$ & $0.78_{-0.34}^{+0.17}$ & $0.06_{-0.03}^{+0.02}$ \\
\hline S29 & 137 & $0.12 \pm 0.03$ & $0.75_{-0.35}^{+0.19}$ & $0.09_{-0.05}^{+0.03}$ \\
\hline K15 & 164 & $0.17 \pm 0.02$ & $0.79_{-0.37}^{+0.17}$ & $0.13_{-0.07}^{+0.03}$ \\
\hline $\mathrm{S} 33 \mathrm{sc}$ & 228 & $0.08 \pm 0.02$ & $0.80_{-0.33}^{+0.16}$ & $0.06_{-0.03}^{+0.02}$ \\
\hline Average & & & $0.77_{-0.34}^{+0.17}$ & \\
\hline
\end{tabular}

Note. - Columns are, from left to right: galaxy name, circular velocity $V_{c}$ in $\mathrm{km} \mathrm{s}^{-1}$, fraction $f_{\text {esc }}$ of emitted photons escaping the galaxy (i.e. not absorbed by dust), fraction $f_{\text {IGM }}$ of these transmitted through the IGM, and resulting observed fraction $f_{\text {obs }}=f_{\text {esc }} f_{\text {IGM }}$. Uncertainties in $f_{\text {esc }}$ represent varying escape fractions in different directions, while uncertainties in $f_{\text {IGM }}$ represent variance in the IGM. Uncertainties in $f_{\text {obs }}$ are calculated as $\left(\sigma_{\text {obs }} / f_{\text {obs }}\right)^{2}=\left(\sigma_{\text {esc }} / f_{\text {esc }}\right)^{2}+\left(\sigma_{\mathrm{IGM}} / f_{\mathrm{IGM}}\right)^{2}$.

leading to less dust and hence larger values of $f_{\text {esc }}$. However, the increased neutral fraction of the IGM scatters a correspondingly higher number of photons out of the line of sight, resulting in a smaller total observed fraction. Figure 8 and Figure 9 shows the impact of the IGM on Ly $\alpha$ profiles at $z \sim 5.8$ and $z \sim 6.5$, respectively, and Table 3 and Table 4 summarizes the obtained fractions. At these redshifts, for the six galaxies for which the calculations have been carried out on average a fraction $f_{\mathrm{IGM}}(z=5.8)=0.26_{-0.18}^{+0.13}$ and $f_{\mathrm{IGM}}(z=6.5)=0.20_{-0.18}^{+0.12}$ of the photons is transmitted through the IGM, consistent with what was obtained by Dijkstra et al. (2007).

\subsection{Probing the Epoch of Reionization}

We now focus on a different topic, namely the RT in the IGM far from the emitting galaxies. Measuring the average transmission in a wavelength interval blueward of the Ly $\alpha$ line for a sample of quasars or other bright sources, one can ascertain the average transmission properties and hence the physical state of the IGM. The interval in which the transmission is calculated should be large enough to achieve good statistics, but short enough that the bluest wavelength does not correspond to a redshift epoch appreciably different from the reddest. Furthermore, in order to probe the real IGM and not the quasar's neighborhood, the upper limit of the wavelength range should be taken to have a value somewhat below $\lambda_{0}$.

Songaila (2004) measured the IGM transmission in the LAFs of a large sample of quasars with redshifts between 2 and 6.5 , in the wavelength interval 1080-1185 $\AA$. In Fig-
TABLE 3

LY $\alpha$ TRANSMISSION FRACTIONS FOR SIX SIMULATED GALAXIES AT $z \sim 5.8$

\begin{tabular}{lcccc}
\hline \hline Galaxy & $V_{c}$ & $f_{\text {esc }}$ & $f_{\text {IGM }}$ & $f_{\text {obs }}$ \\
\hline K15g & 63 & $0.93 \pm 0.02$ & $0.35_{-0.24}^{+0.16}$ & $0.33_{-0.23}^{+0.15}$ \\
K15c & 78 & $0.82 \pm 0.02$ & $0.25_{-0.14}^{+0.11}$ & $0.21_{-0.11}^{+0.09}$ \\
K15b & 88 & $0.46 \pm 0.02$ & $0.23_{-0.14}^{+0.12}$ & $0.11_{-0.06}^{+0.05}$ \\
S29b & 94 & $0.85 \pm 0.01$ & $0.27_{-0.17}^{+0.14}$ & $0.23_{-0.15}^{+0.12}$ \\
S29a & 96 & $0.52 \pm 0.08$ & $0.31_{-0.22}^{+0.14}$ & $0.16_{-0.12}^{+0.07}$ \\
K15a & 108 & $0.17 \pm 0.05$ & $0.16_{-0.11}^{+0.11}$ & $0.03_{-0.02}^{+0.02}$ \\
Average & & & & \\
\hline
\end{tabular}

Note. - Same as Table 2, but for $z=5.8$.

TABLE 4

LY $\alpha$ TRANSMISSION FRACTIONS FOR SIX SIMULATED GALAXIES AT $z \sim 6.5$

\begin{tabular}{lcccc}
\hline \hline Galaxy & $V_{c}$ & $f_{\text {esc }}$ & $f_{\text {IGM }}$ & $f_{\text {obs }}$ \\
\hline K15f & 50 & $0.96 \pm 0.03$ & $0.24_{-0.22}^{+0.18}$ & $0.23_{-0.22}^{+0.17}$ \\
K15e & 62 & $0.91 \pm 0.04$ & $0.24_{-0.22}^{+0.14}$ & $0.22_{-0.20}^{+0.13}$ \\
K15d & 66 & $0.95 \pm 0.02$ & $0.18_{-0.16}^{+0.13}$ & $0.17_{-0.16}^{+0.12}$ \\
K15c & 76 & $0.88 \pm 0.02$ & $0.16_{-0.14}^{+0.07}$ & $0.14_{-0.13}^{+0.06}$ \\
K15b & 89 & $0.83 \pm 0.03$ & $0.20_{-0.17}^{+0.06}$ & $0.17_{-0.14}^{+0.05}$ \\
K15a & 108 & $0.26 \pm 0.09$ & $0.18_{-0.16}^{+0.06}$ & $0.05_{-0.04}^{+0.02}$ \\
Average & & & & \\
\hline
\end{tabular}

Note. - Same as Table 2, but for $z=6.5$.

ure 10 we compare the simulated transmitted fractions with her sample. As is evident from the figure, an "early" reionization, i.e. with $z_{\text {re }}=10$ (Model 1 and 2), yields a slightly too transparent Universe, while a "late" reionization (Model 3) yields a too opaque Universe. Note that the $\log$ scale makes the $z_{\text {re }}=6$ data seem much farther off than the $z_{\text {re }}=10$ data.

\subsection{Convergence test}

In Section 3.4 we stated that the sightlines should initiate at a distance $r_{0}$ from the centers of the galaxies given by their virial radius. Figure 11 displays the cumulative probability distributions of the distance of the last scattering from the galaxy center (calculated with MoCALATA) for different redshifts and mass ranges, demonstrating that in most cases a photon will have experienced its last scattering at the order of $1 r_{\text {vir }}$ from the center of its host galaxy. For increasing redshift, the photons tend to escape the galaxies at larger distances due to the higher fraction of neutral hydrogen, but the change with redshift seems quite slow. Furthermore, at a given redshift the distinction between different galactic 


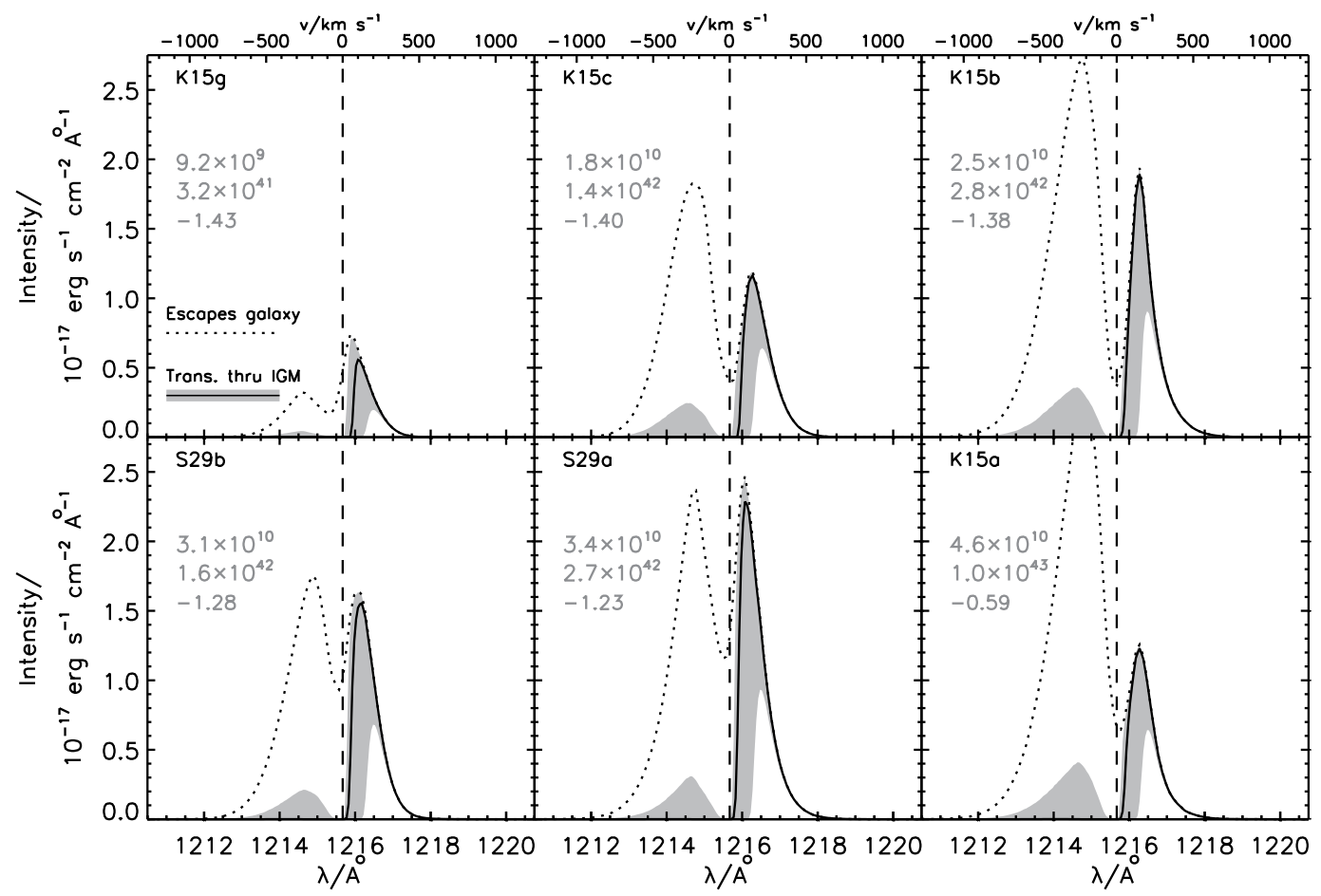

FIG. 8. - Same as Figure 7, but for $z=5.8$.

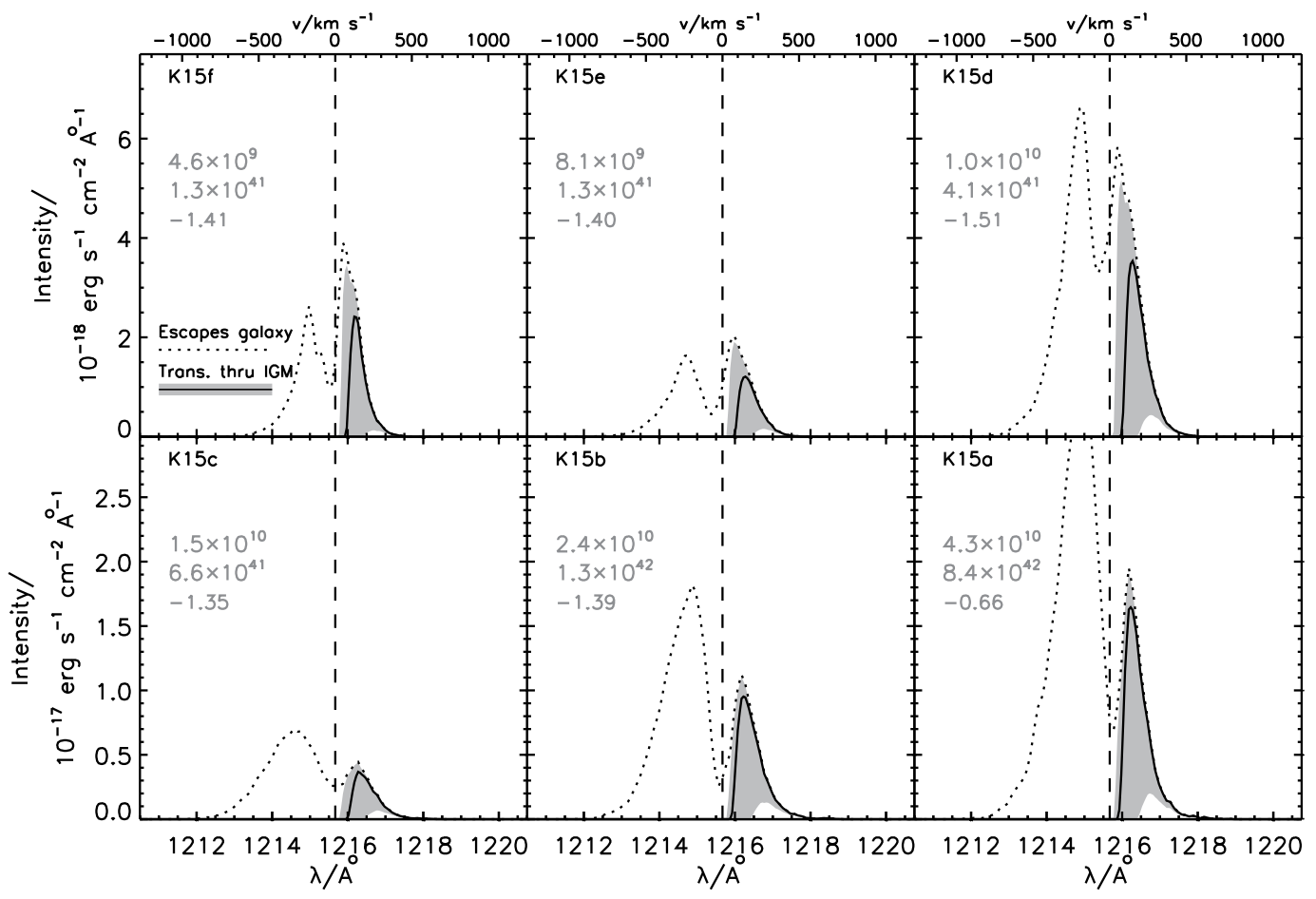

FIG. 9.- Same as Figure 7, but for $z=6.5$. 


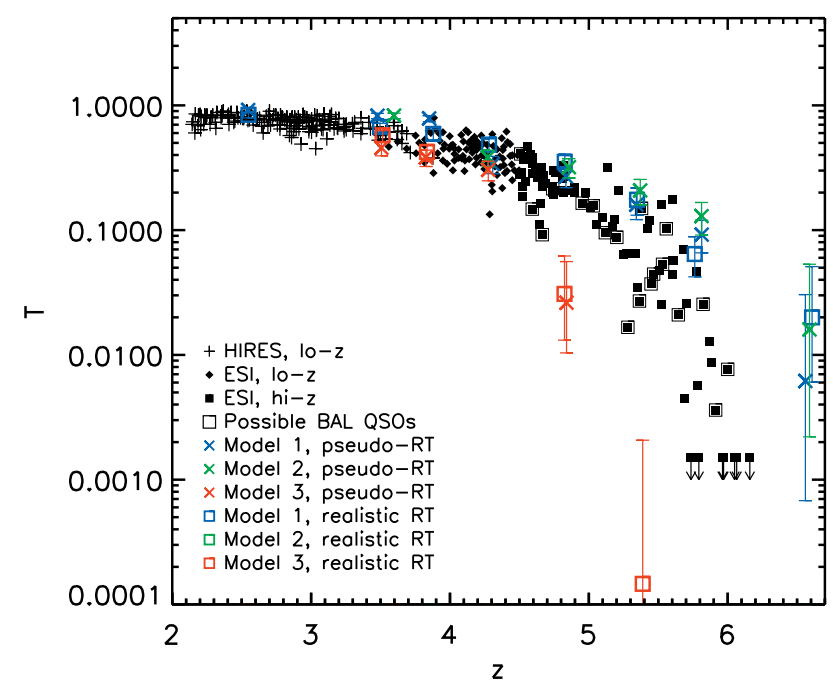

FIG. 10.- Comparison of observations (black data points) and simulations (colored data points) of the transmitted flux blueward of the Ly $\alpha$ line as a function of redshift. The three models are represented by the colors blue, green, and red for model 1,2, and 3 , respectively. To highlight the significance of the improved UV $\mathrm{RT}$, we show both the transmission in the "original" simulation with the "pseudo"-RT (crosses) and with the improved UV RT (squares). For details on the observations see Songaila (2004), from where the data are kindly supplied.

size ranges appears insignificant (as long as $r_{0}$ is measured in terms of $r_{\text {vir }}$ ).

Also shown in Figure 11 are the transmission curves for sightlines initiating at various distances $r_{0}$ from the centers of the galaxies. For very small values of $r_{0}$, a significantly lower fraction is transmitted due to the high density of neutral gas. Around $r \sim r_{\text {vir }}$ the change in $F(\lambda)$ becomes slow, converging to $F(\lambda)$ exhibiting no dip for $r_{0} \rightarrow \infty$.

In summary, scaling $r_{0}$ to the virial radius $r_{\text {vir }}$ of the galaxies allows us to use the same value of $r_{0}=1.5 r_{\text {vir }}$ for all sightlines.

\section{DISCUSSION}

\subsection{The origin of the dip}

In general, the effect of the IGM - even at relatively low redshifts - is to reduce the blue peak of the Ly $\alpha$ line profile. At $z \sim 3.5$, the effect is not strong enough to fully explain the oft-observed asymmetry, but at $z \gtrsim 5$, almost all radiation blueward of the line center is lost in the IGM.

Figures 12 and 13 display for three different epochs the velocity field and the density of neutral hydrogen, respectively, of the IGM associated with the galaxies, taken as an average of all galaxies in the samples, and in all directions. At all redshifts, on average the IGM close to the galaxies recedes at a somewhat slower rate than that given by the pure Hubble flow, slowly converging toward the average expansion rate of the Universe. At the higher redshifts, the IGM in the immediate proximity of the galaxies is characterized by higher velocities, due to outflows generated by starbursts. At $z=3.5$, however, this effect is overcome by the accretion of gas.

Inspecting the dips in Figure 2, the minima are seen to be located at roughly $50 \mathrm{~km} \mathrm{~s}^{-1}$, almost independently of the redshift but becoming slightly broader with increasing $z$. At $z=3.5$, the dip extends all the way out to $300-400 \mathrm{~km} \mathrm{~s}^{-1}$. This corresponds to the central absorption being caused by the IGM within $\sim 150 \mathrm{kpc}$, and the wings of the absorption by the IGM within $\sim 1 \mathrm{Mpc}$. As shown in Figure 13, at $z=3.5$ the density of neutral hydrogen decreases monotonically with distance from the source, and within $\sim 150 \mathrm{kpc} n_{\mathrm{HI}}$ is substantially higher than the cosmic mean. Further away, the density is close to the mean density of the Universe. However, as seen in Figure 12 the recession velocity of the gas continues to lie below that of the average, Universal expansion rate, and in fact does so until approximately $1 \mathrm{Mpc}$ from the source. ${ }^{10}$

Thus, the cause of the suppression of the blue wing of the Ly $\alpha$ line may, at wavelengths close to the line center $(\Delta \lambda \simeq 1 / 2 \AA)$, be attributed to an increased density of neutral hydrogen close to the galaxies, while farther away from the line center $(\Delta \lambda \lesssim 1-1.5 \AA)$, to a retarded Hubble flow.

Zheng et al. (2010a) perform Ly $\alpha$ RT in the IGM at $z=5.7$ through full Monte Carlo simulations, and make a detailed comparison with the observed line profile obtained from simply multiplying the intrinsic line by $e^{-\tau}$, as has been done in previous models (e.g. Iliev et al. 2008). They conclude that neglecting scattering effects severely underestimates the transmitted fraction. While it is certainly true that treating scattering processes as absorption inside the galaxies is only a crude approximation, once the probability of photons being scattered into the line of sight becomes sufficiently small, this approach is quite valid. In their analysis, Zheng et al. (2010a) start their photons in the center of the galaxies, which are resolved only by a few cells (their $d x$ being $\sim 28 \mathrm{kpc}$ in physical coordinates and their fiducial galaxy having $r_{\text {vir }}$ $=26 \mathrm{kpc}$ ). Since the side length of our smallest cells are more than 400 times smaller than the resolution of Zheng et al. (2010a), we are able to resolve the galaxies and their surroundings in great detail, and we are hence able to determine the distance at which the $e^{-\tau}$ model becomes realistic. Moreover, when coupling the IGM RT with Ly $\alpha$ profiles, we use the realistically calculated profile, whereas Zheng et al. (2010a) use a Gaussian set by the galaxies' halo masses. Their line widths $\sigma_{\text {init }}=32 M_{10}^{1 / 3} \mathrm{~km} \mathrm{~s}^{-1}$, where $M_{10}$ is the halo mass divided by $10^{10} h^{-1} M_{\odot}$, thus neglect broadening by scattering. This makes them much smaller than ours, which are typically several hundred $\mathrm{km} \mathrm{s}^{-1}$. Note, however, that our relatively small cosmological volume and the fact that ionizing UV RT is performed as a post-process rather than on the fly may make our density field less accurate than that of Zheng et al. (2010a).

The photons that are scattered out of the line of sight are of course not lost, but rather become part of a diffuse Ly $\alpha$ background. Since more scatterings take place in the vicinity of galaxies, LAEs tend to be surrounded by a low-surface brightness halo, making them look more extended on the sky when comparing to continuum bands

10 Since the cosmological volume is several Mpc across, except for the galaxies lying close to the edge, the absorption takes place before the sightlines "bounce", so in general there is no risk of a sightline going through the same region of space before having escaped the zone causing the dip. 

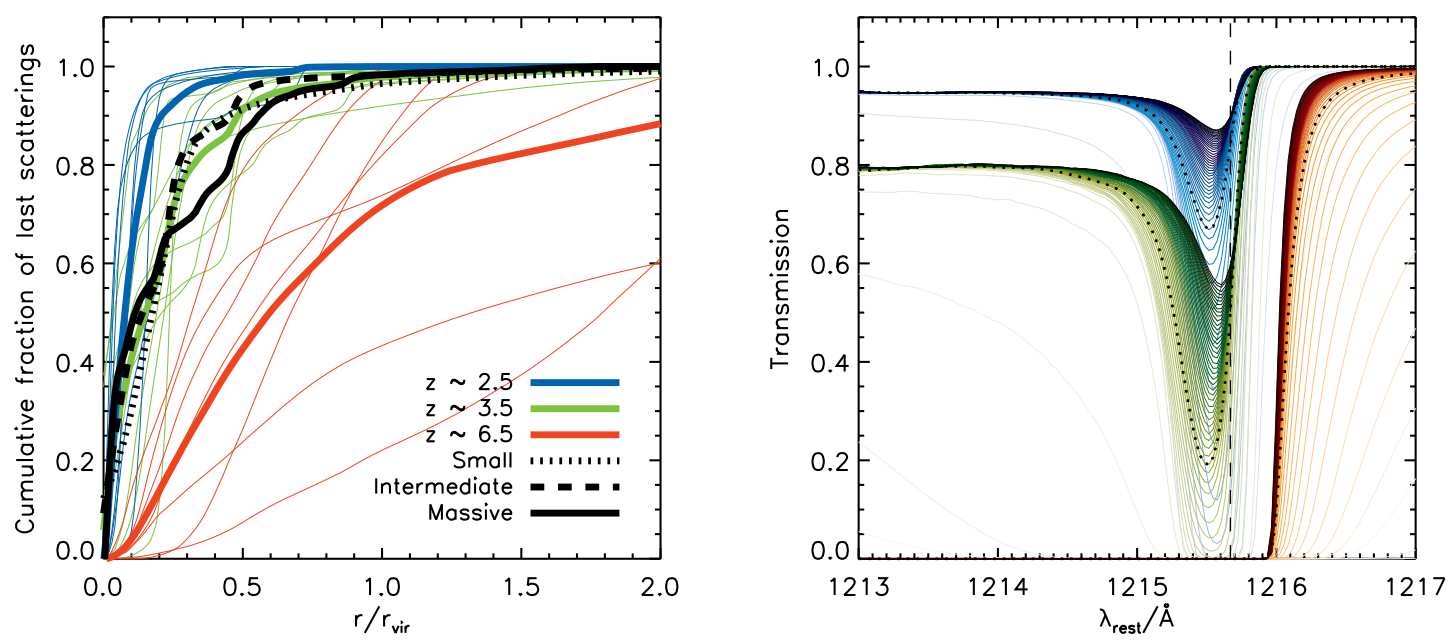

FIG. 11. - Left: Cumulative probability distribution of the distance $r$ from the center of a galaxy at which the last scattering takes place (calculated with the Ly $\alpha$ RT code MoCALATA (Laursen et al. 2009a)). Thin solid lines represent individual galaxies at redshift 2.5 (blue), 3.5 (green), and 6.5 (red), while thick solid colored lines are the average of these. Also shown, in black, are the average of three different size ranges at $z=3.5$; small (dotted), intermediate (dashed), and large galaxies (solid).

Right: Resulting transmission function $F(\lambda)$ for sightlines originating at various distances $r_{0} / r_{\mathrm{vir}}$ from galactic centers, increasing in steps of $0.1 r_{\mathrm{vir}}$ and ranging from $r_{0}=0.1 r_{\mathrm{vir}}$ to $r_{0}=5 r_{\mathrm{vir}}$. The three different redshifts are shown in shades of blue, green, and red, for $z=2.5$, 3.5 , and 6.5, respectively, and the lines go from light shades for low values of $r_{0}$ to dark shades for high values of $r_{0}$. The transmission functions corresponding to $r_{0}=1.5 r_{\text {vir }}$ are shown in black dotted lines. All results are for Model 1.
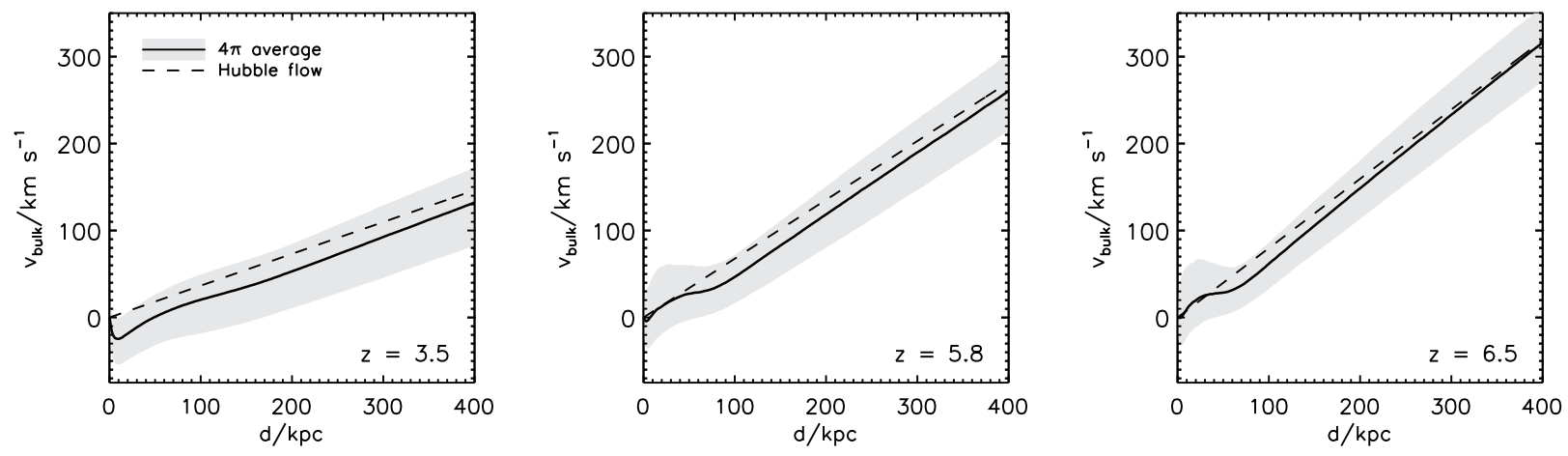

FIG. 12.- Average recession velocity $v_{\text {bulk }}$ of the IGM as a function of proper distance $d$ from the centers of the galaxies in Model 1 (solid black lines, with gray regions indicating the $68 \%$ confidence intervals). At all redshift, the expansion is retarded compared to the pure Hubble flow (dashed line) out to a distance of several comoving Mpc. At high redshifts, however, very close to the galaxies outflows generate higher recession velocities.
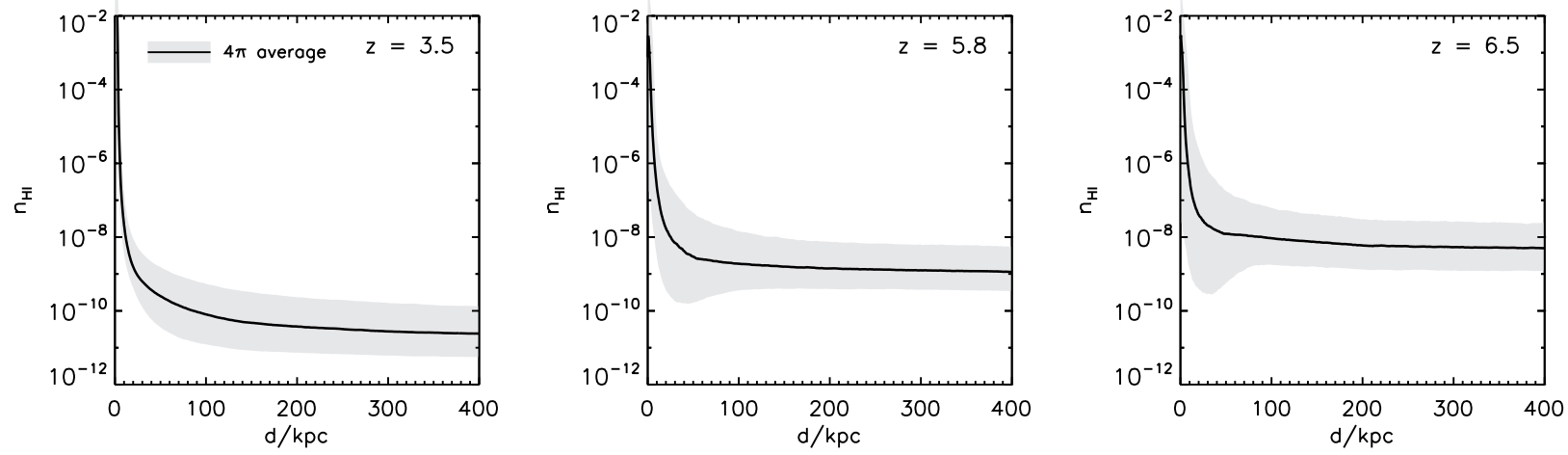

FIG. 13.- Average density $n_{\mathrm{HI}}$ of neutral hydrogen as a function of proper distance $d$ from the centers of the galaxies in Model 1 (solid black lines, with gray regions indicating the $68 \%$ confidence intervals). While in general the density decreases with distance, at high redshifts ionizing radiation reduces $n_{\mathrm{HI}}$ in the immediate surroundings of at least some of the galaxies, as seen by the small dip at $\sim 50$ $\mathrm{kpc}$ in the lower part of the gray zone. 
on the $r \sim 10-100 \mathrm{kpc}$ scale. This has been confirmed observationally (e.g. Møller \& Warren 1998; Fynbo et al. 2001, 2003, but see Bond et al. (2010)), and studied theoretically/numerically (e.g. Laursen \& Sommer-Larsen 2007; Barnes \& Haehnelt 2010; Zheng et al. 2010c). As argued above, part of the cause of the line suppression (mostly in the wings) is due to the IGM up to $\sim 1 \mathrm{Mpc}$ of the source. At this distance, the surface brightness is much lower than observational thresholds, but could be detected by stacking images of LAEs (Zheng et al. 2010c)

As seen in e.g. Figure 3, a large scatter between individual sightlines exists, reflecting the generally quite inhomogeneous IGM. Consequently, one cannot simply use the calculated transmission function for deconvolving observed Ly $\alpha$ lines to obtain the intrinsic line profiles, other than in a statistical sense. With a large sample, however, more accurate statistics on Ly $\alpha$ profiles could be obtained. Calculating the transmission functions as an average of all directions, as we have done in this work, assumes that observed galaxies are randomly oriented in space, i.e. that there is no selection effects making more or less luminous directions pointing toward the observer. For LAEs clustered in, e.g., filaments, the effect of the retarded Hubble flow may be enhanced perpendicular to the filament, making the galaxies more luminous if observed along a filament than perpendicular to it (see Figure 9 in Zheng et al. 2010b).

\subsection{Galactic outflows}

As discussed in the introduction, at high redshifts many galaxies are still in the process of forming and are expected to be accreting gas. In principle, this should result in a blueshifted Ly $\alpha$ profile, but this is rarely seen. Evidently, IGM absorption is unable to always be the cause of this missing blue peak. On larger scales, mass is observed to be conveyed through large streams of gas; the cosmic filaments. Although this has been seen only tentatively on galactic scales, galaxy formation may be expected to occur in a similar fashion. Indeed, numerical simulations confirm this scenario (e.g. Dekel et al. 2009; Goerdt et al. 2010). Very recently, Cresci et al. (2010) reported on an "inverted" metallicity gradient in three $z \sim 3$ galaxies, which they interpret as being due to the central gas having been diluted by the accretion of primordial gas. In contrast to gas accreting through a few narrow streams, outflows are more or less isotropic. Even bipolar outflows have a rather large opening angle, and thus the probability of a sightline towards a galaxy passing through outflowing gas is larger than passing through infalling gas. Thus, it may be expected that more observations are obtained of Ly $\alpha$ profiles lacking the blue peak than lacking the red peak.

The fact that starbursts are needed to generate large outflows also imposes a bias on the observations; even if outflows happen only during relatively short phases in the early life of a galaxy (for LBGs, Ferrara \& Ricotti (2006) found that a typical starburst phase lasts only about $30 \pm 5 \mathrm{Myr}$ ), such galaxies are more likely to appear in surveys, simply because they are more luminous than those with small SFRs.

\subsection{Transmitted fraction of Lya photons}

Even though absorption in the IGM does not alter the line shape drastically at an intermediate redshift of 3.5, it reduces the intensity by roughly one-fourth, as seen from Table 2. This fraction is not a strong function of the size of a galaxy, but since the spectra emerging from larger galaxies tend to be broader than those of smaller galaxies, a comparatively larger part of the small galaxy spectra will fall in the wavelength region characterized by the dip in the transmission function. Hence, on average the IGM will transmit a larger fraction of the radiation escaping larger galaxies.

One-fourth is not a lot, but since it is preferentially blue photons which are lost, the spectrum may become quite skewed when traveling through the IGM. The lost fraction $f_{\text {IGM }}$ is in addition to what is lost internally in the galaxies due to the presence of dust. As mentioned in Section 4.2, dust tends to make the line profile more narrow. For galaxies with no dust, the lines can be very broad. In this case, $f_{\text {IGM }}$ will be slightly higher, since for broad lines a relatively smaller part of the spectrum falls on the dip seen in $F(\lambda)$. Note however that in general distinguishing between dust absorption and IGM effects will be rather tricky, if not impossible (see also Dayal et al. 2010a).

As expected, at higher redshifts the IGM is more opaque to Ly $\alpha$ photon; at $z=5.8$ and $z=6.5$ the intensity was found to be reduced by approximately $75 \%$ and $80 \%$, respectively. At $z=6.5$ the blue wing is completely lost. In most cases this is true also at $z=5.8$. However, as is seen from the gray area representing the $68 \%$ confidence interval, in some cases an appreciable fraction of the blue wing can make it through the IGM.

\subsection{The significance of dust}

The IGM calculations performed in this work are all neglecting the effect of dust. However, dust is mostly (although not completely) confined to the galaxies, especially the central parts, and since by far the greatest part of the distance covered by a given sightline is in the hot and tenuous IGM, one may expect this to be a fair approximation.

This anticipation was confirmed following the dust model of Laursen et al. (2009b). In short, dust density scales with gas density and metallicity, but is reduced in regions where hydrogen is ionized to simulate the effect of dust destruction processes. The factor $n_{\mathrm{HI}} \sigma(\lambda)$ in Equation (2) is then replaced by $n_{\mathrm{H}_{\mathrm{I}}} \sigma(\lambda)+n_{\mathrm{d}} \sigma_{\mathrm{d}}(\lambda)$, where $n_{\mathrm{d}}$ and $\sigma_{\mathrm{d}}(\lambda)$ are the density and cross section of dust, respectively. The resulting decrease in transmission is at the $10^{-4}$ to $10^{-3}$ level.

\section{5. "Early" vs. "late" reionization}

As is evident from Figure 10, although none of the models really fit, the early and late reionization bracket the observations. Model 1 , with $\sigma_{8}=0.74$ provides a somewhat better fit to the observations than Model 2 with $\sigma_{8}=0.9$. Due to the more clumpy structure of the latter, galaxies tend to form earlier, rendering the IGM more free of gas and thus resulting in a slightly more transparent Universe. However, this should not be taken to mean that the lower value of $\sigma_{8}$ is more realistic, since a higher $\sigma_{8}$ could be accounted for by a (slightly) smaller $z_{\text {re. }}$. 
Investigating the LAF is not the only way of probing the EoR. The WMAP satellite detects the effects of the Thomson scattering of cosmic microwave background (CMB) radiation on free electrons. Since the number density $n_{e}$ of free electrons increases as the Universe gets reionized, a signature of the EoR can be obtained by measuring the total optical depth $\tau_{e}$ to Thomson scattering. The latest results (Jarosik et al. 2010) give $\tau_{e}=0.088 \pm 0.015$, implying for their model of the EoR that the Universe was reionized at $z_{\text {reion }} \sim 10-11$.

The optical depth to Thomson scattering in a short interval $d t$ of time is

$$
\begin{aligned}
d \tau & =n_{e}(z) \sigma_{\mathrm{T}} c d t \\
& =n_{e}(z) \sigma_{\mathrm{T}} c \frac{1}{(1+z) H(z)} d z
\end{aligned}
$$

where $\sigma_{\mathrm{T}}$ is the Thomson scattering cross section. That is, given an electron density history, the resulting total $\tau_{e}$ can be calculated by integrating Equation (3).

Figure 14 displays the average comoving number density $n_{e}$ of electrons as a function of redshift, as well as the corresponding total optical depth $\tau_{e}$ of electrons. The results for Model 2 and Model 3 are shown; those for Model 1 lie very close to those of Model 2. Going from larger toward smaller redshifts, both models are seen to be characterized by a roughly constant and very low density of electrons before reionization, then a rapid increase not at, but shortly after $z_{\text {re }}$, and finally a slow decrease, due to subsequent gas cool-out and resulting galaxy formation. The rapid increase marking the EoR lasts approximately 100 Myr. The difference in ionization history for the pseudo- and the realistic UV RT schemes is not critical, although in the latter the creation of ionized bubbles around stellar sources causes a slightly earlier EoR than the pseudo-RT is able to, especially in Model $3\left(z_{\mathrm{re}}=6\right)$.

Also shown in Figure 14 is the corresponding $\tau_{e}$ history that would prevail in the hypothetical case of an instant reionization, where the Universe is fully neutral before, and fully ionized after, some redshift $z_{\text {reion }}$ fixed to make the total $\tau_{e}$ match the value measured by WMAP. In this model, no gas is assumed to be locked up in stars, and $n_{e}$ is thus given by

$$
n_{e}(z)= \begin{cases}0 & \text { for } z>z_{\text {reion }} \\ \frac{\psi \Omega_{b} \rho_{c}(1+z)^{3}}{m_{\mathrm{H}}} & \text { for } z \leq z_{\text {reion }},\end{cases}
$$

where $\psi=0.76$ is the mass fraction of hydrogen, $\Omega_{b}=0.046$ is the baryonic energy density parameter (Jarosik et al. 2010), $\rho_{c}$ is the critical density of the Universe, and $m_{\mathrm{H}}$ is the mass of the hydrogen atom.

As seen from the figure, not even the early reionization model is able to reproduce the optical depth probed by WMAP. However, for a model with an even earlier EoR, the transmission $\mathcal{T}$ of the IGM would be even farther off the observational data, as seen from Figure 10. In general, there seems to be a significant disagreement between the WMAP results and the QSO results concerning the redshift of the EoR. Many authors have tried to resolve this apparent discrepancy, e.g. Wyithe \& Loeb (2003) and Cen (2003) who considered a double reionization, first at $z \sim 15-16$ and later at $z \sim 6$.

Notice nevertheless that the total $\tau_{e}$ of the $z_{\text {re }}=10$ model is not entirely inconsistent with the WMAPinferred value, being roughly $2 \sigma$ away. Furthermore, since only the total optical depth is measured by WMAP, the exact history of the EoR is obviously less certain. Generally, either an instant reionization must be assumed, or perhaps a two-step function to make the EoR slightly extended, and possibly with an additionally step at $z \sim 3$ to account for helium reionization.

The above results are at odds with the interpretation of the CMB EE polarization maps as showing that the reionization of the Universe was complete at $z=10.5$ (Jarosik et al. 2010), regardless of which model is assumed for the EoR. However, many factors enter the conversion of the polarization maps into an optical depth, and the model of this EoR may be too simplistic. Compared to WMAP, the recently launched Planck ${ }^{11}$ satellite has a much higher resolution and sensitivity; when Planck data become available, these issues may be solved as much tighter constraints can be put on parameters like $\tau_{e}$ (Galli et al. 2010).

\section{SUMMARY}

Simulating sightlines through cosmological hydrosimulations, we have investigated the transmission through the IGM of light in the vicinity of the Ly $\alpha$ line. The high resolution of the cosmological simulations combined with the adaptive gridding for the RT allows us to probe the velcity field around the galaxies in great detail. While earlier studies (Zheng et al. 2010a) have shown that this approach of simply multiplying an $e^{-\tau}$ factor on the intrinsically emitted $\operatorname{Ly} \alpha$ line is a poor approximation for the observed line profile and transmitted fraction, we have argued that when the circumgalactic environs are sufficiently resolved and combined with realistically calculated intrinsic Ly $\alpha$ lines, this approach should be valid. Special emphasis was put on how Ly $\alpha$ line profiles emerging from high-redshift galaxies are reshaped by the surrounding IGM. In general a larger fraction of the blue side of the line center $\lambda_{0}$ is lost as one moves toward higher redshifts. At $z \gtrsim 5$, almost all of the light blueward of $\lambda_{0}$ is lost, scattered out of the line of sight by the high neutral fraction of hydrogen. However, even at relatively low redshift more absorption takes place just blueward $\lambda_{0}$. A transmission function $F(\lambda)$ was calculated, giving the fraction of light that is transmitted trough the IGM at various epochs. At all redshifts where some of the light blueward of $\lambda_{0}$ is transmitted (i.e. at redshifts below $\sim 5.5$ ), a significant dip with a width of the order $1 \AA$ is seen. The origin of this dip is a combination of an increased density of neutral hydrogen and a retarded Hubble flow in the vicinity of the galaxies.

This extra absorption may in some cases be the reason that the blue peak of an otherwise double-peaked Ly $\alpha$ profile is severely reduced, or lacking. Nevertheless, it is not sufficient to be the full explanation of the numerous observations of $z \sim 2-4$ Ly $\alpha$ profiles showing only the red peak. The outflow scenario still seems a credible interpretation.

Combining the inferred transmission functions with simulated line profiles, the fraction of Ly $\alpha$ photons that are transmitted through the IGM at $z \sim 3.5,5.8$, and 6.5 was computed, and was found to be $f_{\mathrm{IGM}}(z=3.5)=$ $0.77_{-0.34}^{+0.17}, f_{\mathrm{IGM}}(z=5.8)=0.26_{-0.18}^{+0.13}$, and $f_{\mathrm{IGM}}(z=$

\footnotetext{
11 http://www.rssd.esa.int/Planck/
} 

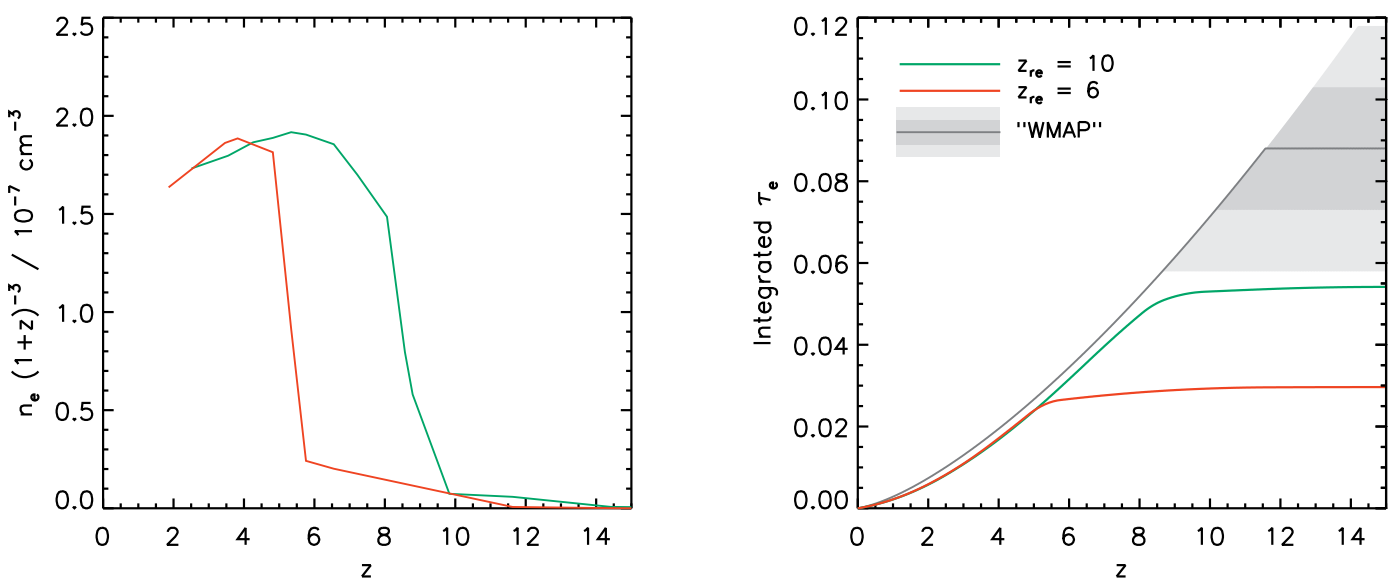

FIG. 14. - Left: Volume averaged, comoving electron density $n_{e} /(1+z)^{3}$ as a function of redshift $z$ for Model $2($ green $)$ and $3($ red $)$ which have $z_{\mathrm{re}}=10$ and $z_{\mathrm{re}}=6$, respectively. Both models are characterized by a quite sharp increase in $n_{e}$ shortly after the onset of the UVB. For Model 2 the EoR is seen to take place around $z \sim 8.5$, while for Model the EoR lies at $z \sim 5.5$.

Right: Integrated optical depth $\tau_{e}$ of electrons as a function of redshift $z$ for the two models. The dark gray line with the associated lighter gray $68 \%$ and $95 \%$ confidence intervals indicates the $\tau_{e}$ history required to reach the optical depth measured by WMAP, if an instant reionization is assumed. As expected, none of the models are able to reach the $0.088 \pm 0.015$ inferred from the WMAP results, although the $z_{\mathrm{re}}=10$ model is "only" $\sim 2 \sigma$ away. Since the $n_{e}$ data do not extend all the way to $z=0$, a fiducial value of $n_{e}(z=0)=1.5 \times 10^{-7}$ $\mathrm{cm}^{-1}$ has been used. The exact value is not very imporant, since the proper density at low redshift is very small. At low redshift, the model curves lie slightly below the theoretical curve. This is due to a combination of the models including helium ionization, releasing more electrons, and star formation and gas cool-out, removing free electrons.

$6.5)=0.20_{-0.18}^{+0.12}$, respectively. This is in addition to what is lost internally in the galaxies due to dust. The standard deviations were found to be dominated by sightlineto-sightline variations rather than galaxy-to-galaxy variations.

Considering the average fraction of light far from the line on the blue side transmitted through the IGM, and comparing to the comprehensive set of observations of the LAF by Songaila (2004), we constrain the EoR to have initiated between $z_{\mathrm{re}}=10$ and $z_{\mathrm{re}}=6$, corresponding the having ionized a significant fraction of the Universe around $z \sim 5.5$ and $z \sim 8.5$, respectively. Even though the "early" models of $z_{\mathrm{re}}=10$ produces a slightly too transparent Universe when comparing to the LAF, the optical depth of electrons is too low when comparing to the observations of the CMB by the WMAP satellite, possibly indicating a too simplistic interpretation of the CMB polarization.

We are grateful to Antoinette Songaila Cowie for letting us reproduce her observational data, to Andrea Ferrara for helpful comments on the electron optical depth, to Zheng Zheng for pointing out errors and deficiencies in the first draft, and to the anonymous referee for comments leading to a substantially extended and better paper.

The simulations were performed on the facilities provided by the Danish Center for Scientific Computing.

The Dark Cosmology Centre is funded by the Danish National Research Foundation.

PL acknowledges fundings from the Villum Foundation.

\section{REFERENCES}

Ambarzumian, V. A. 1932, MNRAS, 93, 50

Atek, H., Kunth, D., Hayes, M., Östlin, G., Mas-Hesse, J. M. 2008, A\&A, 488, 491

Barnes, L. \& Haehnelt, M. G. 2010, MNRAS, 403, 870

Bechtold, J., Crotts, A. P. S., Duncan, R. C., \& Fang, Y. 1994, ApJ, 437, L83

Becker, R. H. et al. 2001, AJ, 122, 2850

Bi, H. \& Davidsen, A. F. 1997, ApJ, 479, 523

Bond, N. A., Feldmeier, J. J., Matković, A., Gronwall, C., Ciardullo, R., \& Gawiser, E. 2010, ApJ, 716, L200

Bruscoli, M., Ferrara, A., Marri, S., Schneider, R., Maselli, A., Rollinde, E., \& Aracil, B. 2003, MNRAS, 343, L41

Cen, R. 2003, ApJ, 591, 12

Chandrasekhar, S. 1935, ZAp, 9, 267

Cresci, G., Mannucci, F., Maiolino, R., Marconi, A., Gnerucci, A., \& Magrini, L. 2010 (arXiv:1010.2534)

Dayal, P., Hirashita, H., \& Ferrara, A., 2010, MNRAS, 403, 620

Dekel, A., Birnboim, Y., Engel, G., Freundlich, J., Goerdt, T., Mumcuoglu, M., Neistein, E., Pichon, C., Teyssier, R., \& Zinger, E. 2009, Nature, 457, 451

Dijkstra, M., Haiman, Z., \& Spaans, M. 2006, ApJ, 649, 14
Dijkstra, M., Lidz, A., \& Wyithe, J. S. B. 2007, MNRAS, 377, 1175

Dinshaw, N., Impey, C. D., Foltz, C. B., Weymann, R. J., \& Chaffee, F. H. 1994, ApJ, 437, L87

Djorgovski, S. G., Castro, S. M., Stern, D., \& Mahabel, A. A. 2001, ApJ, 560, L5

Dunkley, J. et al. 2009, ApJS, 180, 306

Fan, X., Carilli, C. L., \& Keating, B. 2006, ARA\&A, 44, 415

Fang, Y., Duncan, R. C., Crotts, A. P. S., \& Bechtold, J. 1996, ApJ, 462, 77

Faucher-Giguère, C.-A., Kereš, D., Dijkstra, M., Hernquist, L., \& Zaldarriaga, M. 2010 (arXiv:1005.3041)

Faucher-Giguère, C.-A., Prochaska, J. X., Lidz, A., Hernquist, L., \& Zaldarriaga, M. 2008, ApJ, 681, 831

Ferrara, A. \& Ricotti, M. 2006, MNRAS, 373, 571

Finkelstein, S. L., Rhoads, J. E., Malhotra, S., Pirzkal, N., Wang, J. 2007, ApJ, 660, 1023

Finkelstein, S. L., Rhoads, J. E., Malhotra, S., Grogin, N., Wang, J. 2008 ApJ, 678, 655

Fynbo, J. P. U., Møller, P., \& Thomsen, B. 2001, A\&A, 374, 443

Fynbo, J. P. U., Ledoux, C., Møller, P., Thomsen, B. \& Burud, I. 2003, A\&A, 407, 147 
Galli, S., Martinelli, M., Melchiorri, A., Pagano, L., Sherwin, B. D., Spergel, D. N. (arXiv:1005.3808)

Gawiser et al. 2006, ApJS, 162, 1

Gnedin, N. Y. 2000, ApJ, 542, 535

Gnedin, N. Y. Kravtsov, A. V., \& Chien, H.-W. 2004, ApJ, 672, 765

Goerdt, T., Dekel, A., Sternberg, A., Ceverino, D., Teyssier, R., \& Primack, J. R. 2010 MNRAS, 407, 613

Gronwall et al. 2007, ApJ, 667, 79

Guaita, L. et al. 2010, ApJ, 714, 255

Gunn, J. E. \& Peterson, B. A. 1965, ApJ, 142, 1633

Haardt, F. \& Madau, P. 1996, ApJ, 461, 20

Harrington, J. P. 1973, MNRAS, 162, 43

Hayes, M., Östlin, G., Atek, H., Kunth, D., Mas-Hesse, J. M., Leitherer, C., Jiménez-Bailón, E., Adamo, A. 2007, MNRAS, 382,1465

Hayes, M., Östlin, G., Schaerer, D., Mas-Hesse, J. M., Leitherer, C., Atek, H., Kunth, D., Verhamme, A., de Barros, S., \& Melinder, J. 2010 (arXiv:1002.4876)

Hernquist, L., Katz, N., Weinberg, D. H., \& Miralda-Escudé, J. 1996, ApJ, 457, L51

Hibon, P., Cuby, J.-G., Willis, J., Clément, B., Lidman, C., Arnouts, S., Kneib, J.-P., Willott, C. J., Marmo, C., McCracken, H. 2010, A\&A, 515, 97

Hui, L., Gnedin, N. Y., \& Zhang, Y. 1997, ApJ, 486, 599

Iliev, I. T., Shapiro, P. R., McDonald, P., Mellema, G., \& Pen, U.-L. 2008, MNRAS, 391, 63

Jarosik, N. et al. 2010, (arXiv:1001.4744)

Kennicutt, R. C., Jr. 1998, ARA\&A, 36, 189

Komatsu, E. et al. 2009, ApJS, 180, 330

Kroupa, P. 1998, MNRAS, 298, 231

Kunth, D., Mas-Hesse, J. M., Terlevich, E., Terlevich, R., Lequeux, J., \& Fall, S. M. 1998, A\&A, 334, 11

Lai, K., Huang, J., Fazio, G., Cowie, L. L., Hu, E. M., \& Kakazu, Y. 2007, ApJ, 655, 704

Laursen, P. \& Sommer-Larsen, J. 2007, ApJ, 657, L69

Laursen, P., Razoumov, A. O., \& Sommer-Larsen, J. 2009, ApJ, 696,853

Laursen, P., Sommer-Larsen, J., \& Andersen, A. C. 2009, ApJ, 704,1640

Lia, C., Portinari, L., Carraro, G. 2002, MNRAS, 330, 821

Lia, C., Portinari, L., Carraro, G. 2002, MNRAS, 335, 864

Lidz, A., Oh, S. P., \& Furlanetto, S. R. 2006, ApJ, 639, L47

Lynds, R. 1971, ApJ, 164, L73

Meiksin, A. 2005, MNRAS, 356, 596

Mesinger, A. 2009 (arXiv:0910.4161)
Miralda-Escudé, J., Cen, R., Ostriker, J. P., \& Rauch, M. 1996, ApJ, 471, 582

Møller, P. \& Warren, S. J. 1998, MNRAS, 299, 611

Neufeld, D. 1990, ApJ, 350, 216

Nilsson, K. K., Møller, P., Möller, O., Fynbo, J. P. U., Michalowski, M. J., Watson, D., Ledoux, C., Rosati, P., Pedersen, K., Grove, L. F. 2007, A\&A, 471, 71

Nilsson, K. K., Tapken, C., Møller, P., Freudling, W., Fynbo, J. P. U., Meisenheimer, K., Laursen, P., Östlin, G. 2009, A\&A, 498, 13

Osterbrock, D. E. 1989, Astrophysics of Gaseous Nebulae and Active Galactic Nuclei Sausalito: University Science Books

Ouchi, M. et al. 2010 (arXiv:1007.2961)

Rauch, M. \& Haehnelt, M. G. 1995, MNRAS, 275, L76

Razoumov, A. O. \& Cardall, C. Y. 2005, MNRAS, 362, 1413

Razoumov, A. O. \& Sommer-Larsen, J. 2006, ApJ, , 651, 81

Razoumov, A. O. \& Sommer-Larsen, J. 2007, ApJ, , 668, 674

Razoumov, A. O. \& Sommer-Larsen, J. 2009, (arXiv:0903.2045)

Rykoff et al. 2009, ApJ, 702, 489

Salpeter, E. 1955, ApJ, 121, 161

Sargent, W. L. W., Young, P. J., Boksenberg, A., \& Tytler, D. 1980, ApJS, 42, 41

Sommer-Larsen, J. \& Fynbo, J. P. U. 2008, MNRAS, 385, 3

Sommer-Larsen, J., Götz, M., \& Portinari, L. 2003, ApJ, 596, 47

Sommer-Larsen, J., Romeo, A. D., \& Portinari, L. 2005, MNRAS, 357,478

Sommer-Larsen, J. 2006, ApJ, 644, L1

Songaila, A. 2004, 127, 2598

Songaila, A. \& Cowie, L. L. 2002, AJ, 123, 2183

Springel, V. \& Hernquist, L., MNRAS, 333, 649

Tilvi, V. et al. 2010, ApJ, 721, 1853

Vanzella et al. 2008, A\&A478, 83

Venemans, B., et al. 2005, A\&A, 431, 793

Verhamme, A., Schaerer, D., \& Maselli, A. 2006, A\&A, 460, 397

Verhamme, A., Schaerer, D., Atek, H., \& Tapken, C. 2008, A\&A, 491,89

Wang, J.-X., Malhotra, S., Rhoads, J. E., Zhang, H.-T., \&

Finkelstein, S. L. 2009, ApJ, 706, 762

Wyithe, J. S. B. \& Loeb, A. 2003, ApJ, 586, 693

Yee, H. K. C. \& De Robertis, M. M. 1991, ApJ, 381, 386

Zhang, Y., Anninos, P., \& Norman, M. L. 1995, ApJ, 453, L57

Zheng, Z., Cen, R., Trac, H., \& Miralda-Escudé, J. 2010a, ApJ, 716,574

Zheng, Z., Cen, R., Trac, H., \& Miralda-Escudé, J. 2010b (arXiv:1003.4990)

Zheng, Z., Cen, R., Weinberg, D., Trac, H., \& Miralda-Escudé, J. 2010c (arXiv:1010.3017) 